\title{
Adhesive liposomes loaded onto an injectable, self- healing and antibacterial hydrogel for promoting bone reconstruction
}

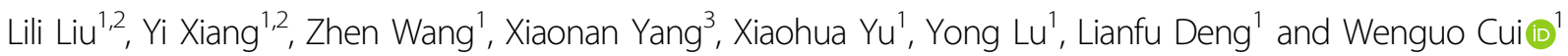

\begin{abstract}
Loading hydrogels with bioactive agents is an important method for expanding the functional application of hydrogels. However, how to improve the local administration and slow release of drugs from a hydrogel is a challenge when using hydrogels loaded with drugs. In this paper, we first developed adhesive liposomes (A-LIP) loaded with BMP-2. Then, we incorporated the A-LIP into PEG hydrogels based on the coordinated cross-linking principle of SHPEG and $\mathrm{Ag}^{+}$, fabricating an injectable, antibacterial and self-healing multifunctional drug delivery system. The adhesive lipo-hydrogel (A-LIP-PEG) fabricated by mixing PEG hydrogels and adhesive liposomes can be locally injected into an osteoporotic fracture and bone marrow cavity, where A-LIP-PEG can release adhesive liposomes that adhere to the bone injury area and promote bone reconstruction. Based on the principle of electrostatic attraction, tissue nonspecific A-LIP were fabricated by grafting octadecylamine onto liposomes. Because of the coordination and crosslinking of thiolated polyethylene (SH-PEG) and $\mathrm{Ag}^{+}$, the A-LIP-PEG showed excellent injectability and self-healing properties; further, because of the presence of $\mathrm{Ag}^{+}$, the A-LIP-PEG showed effective inhibition of S. aureus and Escherichia coli. The liposomes released by the A-LIP-PEG were able to adhere to tissue. In vitro studies showed that ALIP-PEG significantly promoted osteogenic differentiation and had no significant effect on cell proliferation. Compared with common lipo-hydrogel (LIP-PEG), the A-LIP-PEG had better tissue adhesion in vivo, which led to better osteogenic differentiation and faster local bone remodeling of osteoporotic fractures in rats. This research developed a novel hydrogel system with adhesive liposomes to expand the application of hydrogels.
\end{abstract}

\section{Introduction}

Hydrogel represents an attractive scaffold for use in the regeneration of different tissues because of its high water content, good biocompatibility, and tunable mechanical properties $^{1-3}$. Indeed, with the demand for hydrogels in clinical application, numerous kinds of functional hydrogels, such as cell membrane anti-inflammation hydrogel $^{4}$, responsive hydrogel ${ }^{5}$, adhesion hydrogel $^{6}$,

\footnotetext{
Correspondence: Wenguo Cui (wgcui80@hotmail.com)

'Shanghai Key Laboratory for Prevention and Treatment of Bone and Joint Diseases, Shanghai Institute of Traumatology and Orthopaedics, Ruijin Hospital, Shanghai Jiao Tong University School of Medicine, 197 Ruijin 2nd Road, Shanghai 200025, P. R. China

${ }^{2}$ Orthopedic Institute, Soochow University, 708 Renmin Rd., 215006 Suzhou, Jiangsu, China

Full list of author information is available at the end of the article.
}

injectable hydrogel ${ }^{7}$, self-healing hydrogel $^{8}$, and antibacterial hydrogel ${ }^{9}$, have been developed. Many research groups have developed functional hydrogels loaded with nanoparticles, which can show excellent sustained release properties. However, when such hydrogels were implanted into the organism, the bioactive molecules were continuously released, but the flow of body fluid diluted the drug or brought the active molecules to normal tissue areas. This not only reduced the efficacy of active molecules on damaged tissue but also wasted expensive bioactive molecules. If some anti-tumor drugs were carried away from the damage tissue, these drugs could cause great damage to normal tissue. Therefore, it became an urgent issue to develop a hydrogel that increases the retention of bioactive molecules in the target tissue

\section{(c) The Author(s) 2019}

(c) (i) Open Access This article is licensed under a Creative Commons Attribution 4.0 International License, which permits use, sharing, adaptation, distribution and reproduction cc) in any medium or format, as long as you give appropriate credit to the original author(s) and the source, provide a link to the Creative Commons license, and indicate if changes were made. The images or other third party material in this article are included in the article's Creative Commons license, unless indicated otherwise in a credit line to the material. If material is not included in the article's Creative Commons license and your intended use is not permitted by statutory regulation or exceeds the permitted use, you will need to obtain permission directly from the copyright holder. To view a copy of this license, visit http://creativecommons.org/licenses/by/4.0/. 
through adhesion. Among nanoparticles, liposomes have excellent biocompatibility and effective drug delivery of lipophilic, hydrophilic and amphiphilic drugs ${ }^{10}$. However, common liposomes do not adhere to tissue and cannot be retained in target tissue. Therefore, it became an urgent issue to develop a functional hydrogel that was composed of adhesive liposomes. The liposomes are expected to have sustained release and adhere to the tissue for continuous drug delivery.

Mercaptan is considered to be a very effective way to enhance liposome adhesion because the sulfhydryl groups of mercaptan have the unique property of forming disulfide bonds with the cysteine residue of mucin in tissue ${ }^{11}$. However, the disulfide bond between mercaptan liposomes and mucins is reversible, resulting in a short retention time of mercaptan liposomes on the surface of tissues in vivo ${ }^{12}$. Lectins are naturally occurring proteins that can also be used to enhance adhesion properties ${ }^{13}$. However, lectin reversibly binds to the cell surface and has immunogenic and highly toxic properties ${ }^{14,15}$. Therefore, the construction of adhesive liposomes with long-lasting adhesion to tissue and good biocompatibility remains a challenging task. Traditional chitosan derivative-coated adhesive liposomes have the advantages of continuous adhesion and good biocompatibility, but they are not suitable for composite hydrogels. On the one hand, chitosan derivatives were often water-soluble, so in addition to being wrapped in liposomes, most chitosan derivatives contained a large number of chemical bonds that were free in water, which made the preparation of a hydrogel difficult. On the other hand, because chitosan derivatives were on the surface of liposomes but were not directly connected to liposomes, the connection between chitosan derivatives and liposomes is weak. Chitosan derivatives of adhesive liposomes dissociated from hydrogel were easily dissolved and diluted by body fluids, resulting in the loss of adhesion of liposomes. Octadecylamine is an amphiphilic cationic film-forming material that enabled new methods for preparing adhesive liposomes. Based on electrostatic attraction, tissue nonspecific A-LIP were fabricated by grafting octadecylamine onto liposomes. The lipophilic end of octadecylamine was dissolved in the bilayer of a liposome, which linked it firmly to the liposome; this link prevented dilution by the body fluid and kept the liposome adhesive. In addition, natural polymer hydrogels usually contain carboxy and amino groups ${ }^{16-18}$. These groups may form chemical bonds with the surface of liposomes, resulting in the adhesion of liposomes to hydrogel ${ }^{19}$, which are normally difficult to directly adhere to tissue. After the drug was released, liposomes were easily metabolized directly in the tissue. A multiarm thiolated polyethylene glycol hydrogel is rich in sulfhydryl groups and does not contain carboxy and hydroxyl groups ${ }^{20}$, so it does not form chemical bonds with adhesive liposomes. Liposomes released from these hydrogels can continue to release drugs through the adhesion of liposomes to local tissues.

Osteoporosis usually occurs due to a decrease in bone mineral density and a deterioration of bone trabecular microstructure, which leads to an increase in bone vulnerability and results in bone fractures ${ }^{21}$. Osteoporosis caused by bone mass loss leads to local fractures in the hip, vertebral body, and distal forearm. At present, it is difficult to heal bones directly by external fixation because the bone damage was caused by local osteoporosis, which often leads to failure of external fixation of instruments, as well as weak bonding of bone at the interface between the screw and bone tissue ${ }^{22-24}$. Therefore, developing an injectable material for bone regeneration has become an urgent issue for improving bone reconstruction and repair of the local damaged bone cavity (Scheme 1). In this paper, based on the coordination cross-linking principle of $\mathrm{SH}-\mathrm{Ag}$, an injectable, antibacterial and self-healing hydrogel with no active group was developed, and then adhesive liposomes were fabricated that were loaded with BMP-2 through electrostatic attraction. A-LIP-PEG without bonding was directly injected into the osteoporotic fracture area and the bone marrow cavity, and the local adhesive liposomes were released for bone reconstruction in situ. The system achieved the following results: (1) Adhesive drug-loaded liposomes stimulated by electrostatic attraction were fabricated to achieve local adhesive drug delivery. (2) Fabrication of injectable, antibacterial and self-healing PEG hydrogel was shown to directly fill the osteoporotic fracture area and cavity. (3) A-LIP-PEG hydrogel released A-LIP, and A-LIP adhered to damaged tissue, which could increase the retention of bioactive molecules in target tissue, maintain local drug concentration for a long time and reduce the waste of expensive bioactive molecules. Therefore, the hydrogel drug loading system provides a new treatment method for the treatment of bone cavity damage and diseases.

\section{Materials and methods \\ Preparation of liposomes}

A thin film dispersion method was used to prepare adhesive liposomes (A-LIP)-loaded BMP-2 (Lot No. MSA5518011, R\&D Systems Co., Ltd). In short, lecithin (Lot No. S30870, Shanghai Yuanye Biochemical Co., Ltd)/ cholesterol (Lot No. A0369775, Acros Co., Ltd)/octadecylamine (Lot No. L1605024, Aladdin Biotechnology Co., Ltd.) (40:10:2 w/w) was prepared in a phospholipidchloroform solution. Thin films were formed in a round bottom flask by evaporation at $50 \mathrm{r} / \mathrm{min}$ and $60^{\circ} \mathrm{C}$ in a rotating evaporator. Subsequently, the film was hydrated with preheated deionized water containing BMP-2 at $25^{\circ} \mathrm{C}$ and $50 \mathrm{r} / \mathrm{min}$. The product was treated by ultrasound and filtered sequentially through polycarbonate membrane 


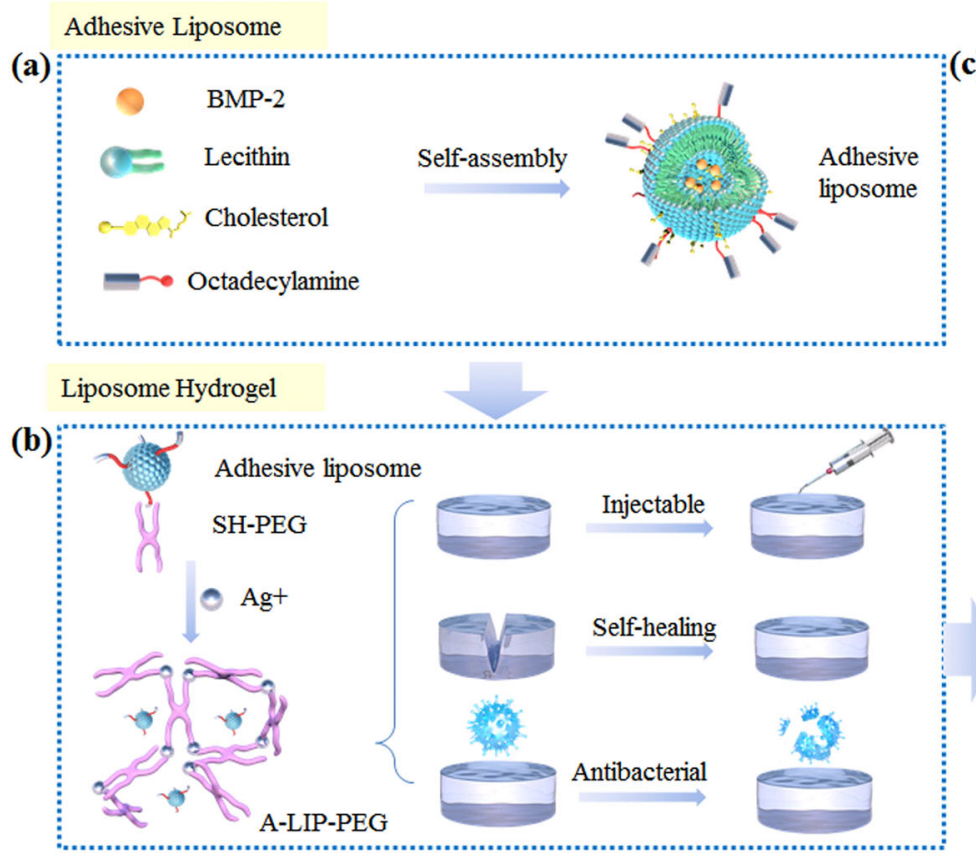

Osteogenesis

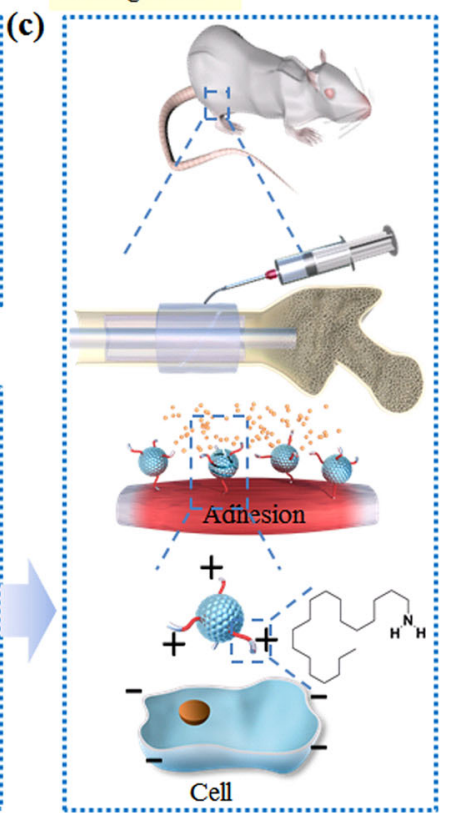

Scheme 1 Schematic illustrations of A-LIP-PEG for stimulating osteogenesis. aSchematic illustration of A-LIP formation. b A-LIP, SH-PEG, and $\mathrm{Ag}+$ constructed by $\mathrm{Ag}-\mathrm{S}$ coordination form an adhesive, liposome-laden, injectable, self-healing, and antibacterial hydrogel (A-LIP-PEG). c A-LIPPEG was locally injected into an osteoporotic fracture and a bone marrow cavity, and the A-Lip released from A-LIP-PEG adhered to the lesion site in situ to release drugs.

filters with pore sizes of $450 \mathrm{~nm}$ and $220 \mathrm{~nm}$ to obtain BMP-2-loaded A-LIP with a BMP-2 concentration of $20 \mu \mathrm{g} / \mathrm{mL}$. The common liposome (LIP) was prepared in a similar manner, but it did not contain octadecylamine. Fluorescent adhesive liposomes (FI-A-LIP) were prepared in a similar manner. In short, lecithin/cholesterol/octadecylamine (40:10:2 w/w) was prepared into phospholipidchloroform solution. Then, $200 \mu \mathrm{L}$ of $1 \mathrm{mg} / \mathrm{mL}$ coumarin- 6 (CAS: 38215-36-0, Sigma-Aldrich Technology Co., Ltd) ethanol solution was added to the phospholipidchloroform solution. The following experimental operation was the same as above except for avoiding light. Common fluorescent liposomes (FI-LIP) were prepared in a similar manner without adding octadecylamine. Briefly, lecithin/cholesterol $(40: 10 \mathrm{w} / \mathrm{w})$ was prepared into phospholipid-chloroform solution. Two hundred microliters of coumarin- 6 ethanol solution at a concentration of $1 \mathrm{mg} / \mathrm{ml}$ was added to the phospholipid-chloroform solution. The following experimental operation was performed as described above. DIR fluorescent adhesive liposomes (DIR-A-LIP) and DIR fluorescent liposomes (DIR-LIP) were prepared by similar methods. Briefly, lecithin/cholesterol/octadecylamine $(40: 10: 2 \mathrm{w} / \mathrm{w})$ was prepared into phospholipid-chloroform solution. Then, $100 \mu \mathrm{g}$ DIR (MB12482, Meilunbio, China) was added to the phospholipid-chloroform solution. The following experimental operation was performed as described above.

\section{Preparation of lipo-hydrogels}

SH-PEG (Lot No. TZQ09095, Creative PEGWorks Co., Ltd) $(25 \mathrm{mg})$ was weighed and dissolved in $100 \mu \mathrm{L}$ A-LIP. Then, $75 \mu \mathrm{L} \mathrm{AgNO}_{3}$ (Lot No. 20170808, Sinopharm Chemical Reagent Co., Ltd.) in an aqueous solution $(0.1 \mathrm{M})$ was diluted with $100 \mu \mathrm{L}$ A-LIP. The liquid in the two tubes was mixed, and the two solutions formed a transparent A-LIP-PEG after a few seconds. Common lipo-hydrogel (LIP-PEG) was prepared in a similar manner except that SH-PEG $25 \mathrm{mg}$ was dissolved in $100 \mu \mathrm{L}$ LIP. FI-A-LIP-PEG and FI-LIP-PEG were prepared in a similar manner. DIR fluorescent adhesive lipo-hydrogel (DIR-A-LIP-PEG) and DIR fluorescent lipo-hydrogel (DIR-LIP-PEG) were prepared in a similar manner.

\section{Physicochemical characterization of liposomes}

A-LIP and LIP solutions were diluted to the appropriate concentrations. The particle size distribution, polydispersity index and zeta potential were measured by a dynamic light scattering particle size dispersion instrument (DLS, Zetasizer, Malvern, Nano-ZS90). To determine the encapsulation efficiency of BMP-2, a Millipore instrument $(\mathrm{Mw}=100,000)$ was used to separate the unloaded drugs. A-LIP and LIP were added to the ultrafiltration device and then centrifuged at $5000 \mathrm{rpm}$ for $40 \mathrm{~min}$. The liquid at the bottom of the tube was collected, and the amount of free drug, $C_{\mathrm{f}}$, was determined. 
Then, 10-times the volume of methanol was added to ALIP and LIP, and the total drug dose, $C_{t}$, was determined. Copper mesh was placed on the filter paper, and $20 \mu \mathrm{L} \mathrm{A-}$ LIP and LIP solution were dripped onto the copper mesh. When the solvent on the copper mesh was dried, the morphology of liposomes was observed by transmission electron microscopy (FEI Tecnai $\mathrm{G} \leq 20$ ).

$$
\mathrm{EE} \%=100-\frac{C_{\mathrm{f}}}{C_{\mathrm{t}}} \times 100
$$

\section{In vitro liposome adhesion test}

The adhesion of liposomes was evaluated following a previously published protocol ${ }^{25}$. CM-Dil cell membrane fluorescent dye (CAS:180854-97-1. Yeasen Biotechnology Co., Ltd.) $(10 \mu \mathrm{g} / \mathrm{mL})$ was incubated with BMSCs for $60 \mathrm{~min}$. After incubation, BMSCs were washed twice with PBS. To evaluate the adhesion of liposomes, $1 \mathrm{~mL}$ FI-ALIP and FI-LIP were incubated in a 6-well plate coated with a monolayer of rat bone marrow mesenchymal stem cells (BMSCs) for $2 \mathrm{~h}$. After incubation, BMSCs were washed twice with PBS. The purpose of washing with PBS was to remove non-adhesive fluorescent liposomes. Then, DAPI was added for fluorescence microscope imaging (ZEISS, Germany). The intensity of the fluorescent signal was quantified by ImageJ (National Institutes of Health, USA).

\section{Properties of lipo-hydrogels}

The freeze-dried lipo-hydrogels were evaluated by scanning electron microscopy (SEM). The layers of ALIP-PEG and LIP-PEG, which were freeze-dried for 2 days, were fixed on a conductive plate and sprayed with gold for $60 \mathrm{~s}$ by ion sputtering (SC7620, Quorum Technologies, UK). Finally, images were collected by scanning electron microscopy (SEM, S-4800-Hitachi, Tokyo, Japan). In addition, heroic red ink (Shanghai Hero Co., Ltd) was used to stain A-LIP-PEG, which were then injected using syringes with a $1-\mathrm{mL}$ needle to illustrate the injectivity of A-LIP-PEG. To demonstrate their ability to self-heal, A-LIP-PEG was divided into two parts (green and red), and then the surfaces of the two parts were assessed for the ability to rejoin over a 20 -min period.

\section{Rheology test of lipo-hydrogels}

The rheological properties of hydrogels were determined on a strain-controlled rheometer (HAAKE RheoStress 6000, Thermo Scientific). A total of $200 \mu \mathrm{L}$ lipo-hydrogel was placed between parallel plates with a gap of $2 \mathrm{~mm}$. To measure the storage modulus $\mathrm{G}^{\prime}$ and loss modulus $\mathrm{G}^{\prime \prime}$, the frequency was set at $10 \mathrm{~Hz}$, and the dynamic strain scanning was from 4 to $100 \%$.

\section{Physicochemical characterization of lipo-hydrogels}

The release behavior of A-LIP-PEG and LIP-PEG was evaluated. The samples were immersed in PBS at $37^{\circ} \mathrm{C}$ and incubated at room temperature while mixing at $120 \mathrm{rpm}$. The release medium was taken at a set time point, and the amount of released BMP-2 was measured using an ELISA kit (Lot No. A110890445, Multisciences Biotechnology Co., Ltd.). Infrared (IR) spectra were measured by FT-IR (Vertex 70, Bruker, Germany). To explore the swelling properties of lipo-hydrogels, the gels were immersed in deionized water at $37^{\circ} \mathrm{C}$ for $24 \mathrm{~h}$ to achieve an equilibrium of swelling, and then they were weighed to determine their initial mass. In addition, to explore the degradation properties of lipo-hydrogels, the gels were kept in deionized water at $37^{\circ} \mathrm{C}$ under mild oscillation, and the remaining mass was recorded periodically at set time points.

\section{Adhesion test of liposomes released from lipo-hydrogels}

To evaluate the adhesion of liposomes released from lipo-hydrogels, $550 \mu \mathrm{L}$ FI-A-LIP-PEG and FI-LIP-PEG were placed in a $5-\mathrm{mL}$ EP tube, $700 \mu \mathrm{L}$ deionized water was added, and the tube was placed on a shaking table for $4 \mathrm{~h}$ to release FI-A-LIP and FI-LIP, respectively. Deionized water without A-LIP and LIP was used as the control group. Then, a monolayer of rat bone marrow mesenchymal stem cells (BMSCs) was washed twice with PBS at $37^{\circ} \mathrm{C}$, and then CM-Dil cell membrane fluorescent dye $(10 \mu \mathrm{g} / \mathrm{mL})$ was incubated with BMSCs for $60 \mathrm{~min}$. After the dye was removed, BMSCs were washed twice with PBS. The released FI-A-LIP-PEG and FI-LIP-PEG solutions were added to the 6-well plate of BMSCs and incubated $37^{\circ} \mathrm{C}$ for $2 \mathrm{~h}$. After incubation, BMSCs were washed twice with PBS to remove non-adhesive fluorescent liposomes, and DAPI was added for fluorescence microscope imaging (ZEISS, Germany). The intensity of the fluorescent signal was quantified by ImageJ. To evaluate the adhesion of liposomes released from lipohydrogels in vivo, $550 \mu \mathrm{L}$ DIR-A-LIP-PEG and DIR-LIPPEG were placed in $5 \mathrm{~mL}$ EP tubes, $700 \mu \mathrm{L}$ deionized water was added, and the tubes were placed on a shaking table for $4 \mathrm{~h}$ to release DIR-A-LIP and DIR-LIP, respectively. Then, DIR-A-LIP and DIR-LIP were administered intraperitoneally to male C57BL/6 mice for 6 weeks. Each mouse was intraperitoneally injected with $100 \mu \mathrm{L}$ of $5 \mathrm{mg} /$ $\mathrm{ml}$ liposomes. The fluorescence distribution was detected by in vivo fluorescence imaging (IVIS Lumina $0 \mathrm{R}$ III, PerkinElmer, USA) at the time points of 3 days.

\section{Antibacterial performance of lipo-hydrogels}

Staphylococcus aureus and Escherichia coli were cultured in sterilized LB at $37{ }^{\circ} \mathrm{C}$ overnight in a rotating oscillator. The bacterial suspension was diluted to the required concentration before use. The Kirby Bauer 
technique was used to test the antibacterial activity of the lipo-hydrogels ${ }^{26,27}$. In short, $100 \mu \mathrm{L}$ S. aureus and Escherichia coli (OD at $600 \mathrm{~nm}$ ) were dispersed on LB agar plates. Next, $100 \mu \mathrm{L}$ A-LIP-PEG and LIP-PEG were placed on agar plates and incubated at $37^{\circ} \mathrm{C}$ for $24 \mathrm{~h}$. After incubation, bacterial inhibition halos around the lipo-hydrogel samples were observed, and the diameter of the bacterial inhibition halos was measured. To observe the live/dead status of the bacteria experimentally, a fluorescence-based cell live/dead assay was carried out $^{28,29}$. A total of $100 \mu \mathrm{L}$ A-LIP-PEG and LIP-PEG were immersed in $1000 \mu \mathrm{L}$ bacterial culture medium with a bacterial concentration of approximately $1 \times 10^{8}$ CFUs/ $\mathrm{mL}$ (CFU: colony forming unit). The mixture was then incubated for $12 \mathrm{~h}$. The bacterial solution without samples was used as the control group. Then, the bacteria treated with the samples were stained simultaneously with SYTO9 and PI (LIVE/DEAD Baclight Bacterial Viability Kit, Thermo Fisher Scientific, for microscopy and quantitative assays) for $15 \mathrm{~min}$, and then they were rinsed with PBS three times. After the plate was dry, images were taken on a fluorescence microscope (ZEISS, Germany). Green fluorescence indicated live bacteria, and red fluorescence showed dead bacteria. To quantify the antibacterial activity of lipo-hydrogel, $100 \mu \mathrm{L}$ A-LIP-PEG and LIP-PEG were added to a $1.5-\mathrm{mL}$ LB solution for $12 \mathrm{~h}$. Ten microliters of lipo-hydrogel extract was mixed with $990 \mu \mathrm{L}$ bacterial culture medium with a bacterial concentration of approximately $1 \times 10^{8} \mathrm{CFUs} / \mathrm{mL}$ (CFU: colony forming unit). The mixture was then incubated at $37^{\circ} \mathrm{C}$ overnight. Then, $100 \mu \mathrm{L}$ of the mixture was coated on an agar plate. After incubation for $24 \mathrm{~h}$, the number of colonies on the agar plate was counted, and the bacterial inhibition ratios of lipo-hydrogels were calculated. The agar plate treated with LB containing only bacteria was used as a control.

$$
\text { Inhibition } \operatorname{ratio}(\%)=100-\frac{N_{\mathrm{t}}-N_{0}}{N_{\mathrm{con}}-N_{0}} \times 100
$$

$N_{0}$ was the seeded bacteria concentration in LB medium (CFUs $/ \mathrm{mL}$ ), $N_{\mathrm{t}}$ and $N_{\text {con }}$ were the bacterial concentration $24 \mathrm{~h}$ after incubation of lipo-hydrogels group and pure LB medium control group, respectively.

\section{Assessment of biocompatibility}

Proliferation of rat bone marrow mesenchymal stem cells (BMSCs) was measured by CCK- 8 assay (Lot No. C0038, Beyotime Biotechnology Co., Ltd.). Extracts from cells treated with A-LIP-PEG and LIP-PEG were collected to investigate the effect of lipo-hydrogels on the viability of BMSCs. BMSCs cultured in Alpha MEM (Lot No. AD16700296, HyClone Co., Ltd.) were used as a control group. BMSCs (3000 cells/well) were cultured in 96-well plates to generate the extract from each group. The activity of BMSCs was detected by CCK- 8 assays at 1,3 , and 5 days of treatment. The extracts of cells treated with A-LIP-PEG and LIP-PEG were collected, and the BMSCs cultured in only medium were used as the control group. BMSCs (30,000 cells/well) were cultured in 24-well plates with the extract of each group. After 2 days of culture, images of live/dead assays were collected by fluorescence microscopy (ZEISS, Germany). In the live/dead experiment, the cells in the A-LIP-PEG group, the LIP-PEG group and the control group were seeded on a 24-well plate. To further evaluate the compatibility of lipohydrogels, BMSCs (30,000 cells/well) were seeded directly onto a lipo-hydrogel disk in a 24-well culture plate. One day later, the cells were stained, and the morphology of the cells seeded on the surface of the cultured lipohydrogel was observed by fluorescence microscopy. In the cell morphology experiment, the cells were seeded on ALIP-PEG hydrogel, LIP-PEG hydrogel and a 24-well plate, which are referred to as the A-LIP-PEG group, the LIPPEG group and the control group, respectively.

\section{In vitro osteogenesis study}

Mouse bone marrow mesenchymal stem cells (BMSCs) (30,000 cells/well) were inoculated into 24-well Transwell plates and cultured in Alpha MEM. After $24 \mathrm{~h}, 50 \mu \mathrm{L}$ ALIP-PEG and LIP-PEG were placed in the upper compartment of the Transwell plate, and BMSCs without treatment were used as the control group. Osteogenic induction medium (Lot No. RASMX-90021, Cyagen Biotechnology Co., Ltd.) was used to culture cells instead of Alpha MEM. After $7 \mathrm{~d}$, the cells were washed with PBS 3 times, fixed with anhydrous ethanol at room temperature for $30 \mathrm{~min}$, washed with deionized water 3 times and treated with alkaline phosphatase staining solution (Lot No. C3206, Beyotime Biotechnology Co., Ltd.). After incubating for $30 \mathrm{~min}$ at room temperature in darkness, the 24-well plates were washed with distilled water, and images were collected by microscopy. Alizarin red S staining was performed to show mineralized nodules in cultured BMSCs. BMSCs (30,000 cells/well) were inoculated into 24-well Transwell plates and cultured in Alpha MEM. After $24 \mathrm{~h}, 50 \mu \mathrm{L}$ A-LIP-PEG and LIP-PEG were placed in the upper compartment of the Transwell plates, and BMSCs without treatment were used as the control group. Osteogenic induction medium was used instead of Alpha MEM. After 14 and 21 days, the cells were washed three times with PBS, fixed at room temperature with a $4 \%$ paraformaldehyde solution for $30 \mathrm{~min}$, washed with deionized water 3 times and incubated with alizarin red $S$ staining solution (Lot No. MKCC2711, Sigma-Aldrich Co., Ltd.). After $10 \mathrm{~min}$ at room temperature, the 24-well plates were washed with distilled water, and images were collected by microscopy. 


\section{Immunocytochemistry}

After 3 weeks of culture, the osteogenic differentiation of BMSCs was observed by immunocytochemical staining. Cells were fixed in $4 \%$ paraformaldehyde, permeabilized with $0.3 \mathrm{v} / \mathrm{v} \%$ Triton X-100 (Lot No. SLBV4122, Sigma Biotechnology Co., Ltd.) and blocked overnight with 5\% bovine serum albumin (BSA). Cells were incubated with OCN antibody (Lot No. IFS0517081, Bio-Techne Biotechnology Co., Ltd.) (1:25) overnight at $4{ }^{\circ} \mathrm{C}$. Alexa Fluor0R 488-conjugated AffiniPure donkey anti-mouse IgG (Lot No. 134982, Jackson Immuno Biotechnology Co., Ltd.) (1:500) was incubated with cells at $37^{\circ} \mathrm{C}$ for $1 \mathrm{~h}$. The samples were then stained with TRITC-phalloidin (Lot No. 40734ES75, Yeasen Biotechnology Co., Ltd.) (1:200) for $30 \mathrm{~min}$ to observe filamentous actin (F-actin). The nuclei were stained with DAPI (Lot No. ab104139, Abcam Biotechnology Co., Ltd.), and the cells were examined under a fluorescence microscope.

\section{Osteogenic gene expression}

The hydrogels and BMSCs were incubated in osteogenic extract medium for 5 days, and BMSCs without hydrogels were used as the control group. Next, total RNA was isolated using a Total RNA Kit I (Thermo Scientific), and it was reverse transcribed to generate cDNA with a PrimeScript RT Master Mix (TaKaRa, Japan). RT-PCR analysis was performed on a Bio-Rad RT-PCR system. The gene expression of osteopontin (OPN), osterix (OSX), and type 1 collagen (COL 1$)$ was measured, and the gene expression level of $\beta$-actin was used for data normalization. The primer sequences for the abovementioned genes are listed in Table S1.

\section{Establishment of osteoporotic femoral fractures in vivo}

Animal experiments were approved by the Experimental Animal Welfare and Ethics Committee of Soochow University. Female and male 8-week-old male Sprague Dawley rats were used in the study, and the animals were maintained under specific pathogen-free conditions. Animal experiments were conducted in accordance with international ethics guidelines and the National Institutes of Health Guide concerning the Care and Use of Laboratory Animals. Twelve weeks after ovariectomy, osteoporosis rat models were randomly divided into three groups: A-LIP-PEG, LIP-PEG and control $^{30}$. Under general anesthesia with chloralhydrate (Lot No. E601BA0047, BBI Life Sciences Biotechnology Co., Ltd.), a proximal incision was made in the lateral femur from the distal end of the lateral condyle to the greater trochanter. The femur was exposed by separating the gluteal finger muscle from the biceps femoris. A transverse incision was made in the middle of the femur with a ring saw. The right medial patellar incision was made on the right knee, and the patella was dislocated to expose the femoral condyle ${ }^{31}$. The metal bone needle $(1.5 \times 230$, tecenet, Shanghai Youke Orthopedics Co., Ltd.) was introduced into the canal and moved to the greater trochanter in a retrograde intramedullary manner. Then, $100 \mu \mathrm{L}$ of lipo-hydrogel was injected into the osteoporotic fracture area and the bone marrow cavity. Muscles were closed with interruptible absorbable sutures followed by closure of the $\operatorname{skin}^{32}$.

\section{Evaluation of osteoporotic fracture healing}

At 2, 4, and 8 weeks after the operation, the rats were anesthetized with chloral hydrate, and the right femur of each group was photographed by X-ray photography (E305451006, D ë RR DENTAL, Germany) under the conditions of $50 \mathrm{kV}, 2.5 \mathrm{~ms}$. Femoral callus formation and fracture healing were observed. To quantitatively analyze osteoporotic fracture healing, the specimens were scanned using a Micro-CT system (SkyScan1176, SkyScan, Aartselaar, Belgium) after the bone needles were removed. Then, 3D digital images were constructed for each sample using analysis software. The volume of interest (VOI) consisted of 200 slices above and below the fracture line. The following parameters were measured and calculated: callus bone mass (BV), callus bone volume fraction (BV/ $\mathrm{TV})$, trabecular thickness (Tb. Th) and trabecular separation (Tb. Sp). These samples were immersed in $10 \%$ EDTA for $\sim 4$ weeks for decalcification. Then, the specimens were embedded in paraffin and cut into $5 \mu \mathrm{m}$ sections. To further evaluate the healing of fractures, specimens were stained with hematoxylin and eosin (HE), Masson trichrome, safranin $\mathrm{O}$, and fast green.

\section{Statistical analysis}

Statistical analysis was evaluated using one-way analysis of variance (ANOVA), which was followed by Tukey's multiple comparison test to evaluate differences between groups. All data were processed using IBM SPSS Statistics 23 for Windows. Data are presented as the mean \pm standard deviation (SD). A probability value of $p<0.05$ was considered statistically significant.

\section{Results and discussion}

Adhesive liposomes were composed of phospholipids, cholesterol and octadecylamine (Fig. 1a). Phospholipids were the main materials used in the formation of liposomes, and the role of cholesterol was to stabilize the liposomes. Octadecylamine converted the negative potential of liposomes to a positive potential. The morphology, particle size distribution, and zeta potential of ALIP and LIP were characterized by transmission electron microscopy (TEM) and dynamic light scattering (DLS). The average particle sizes of A-LIP and LIP were 268.4 \pm $19.0 \mathrm{~nm}$ and $243.4 \pm 16.4 \mathrm{~nm}$, respectively, and the polydispersity indexes (PDI) were $0.249 \pm 0.011$ and $0.209 \pm$ 
(a)

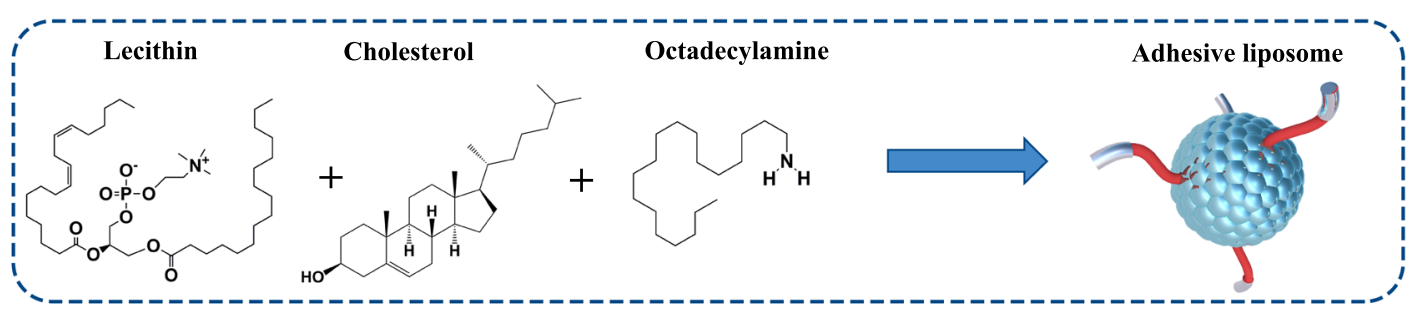

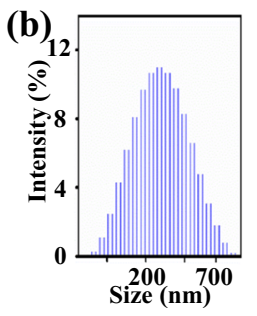

(f)

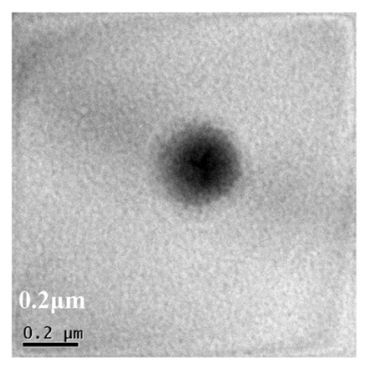

(c)

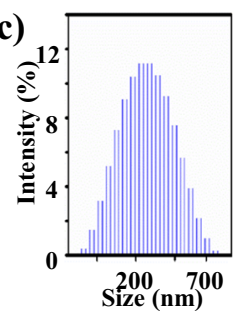

(g)

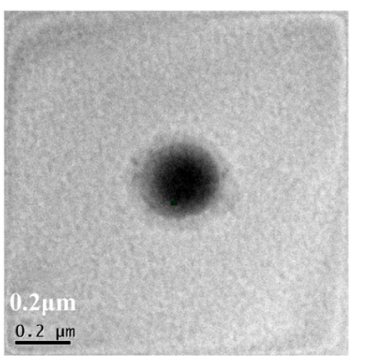

(d)

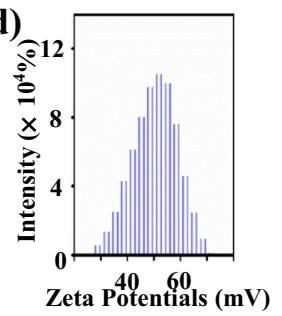

(e)

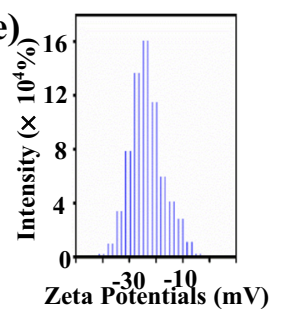

(h)

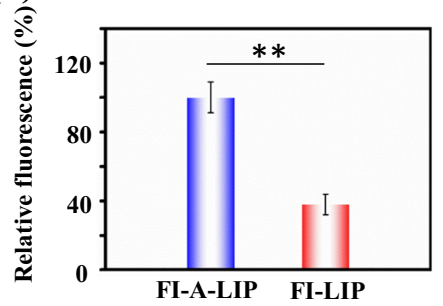

(i)
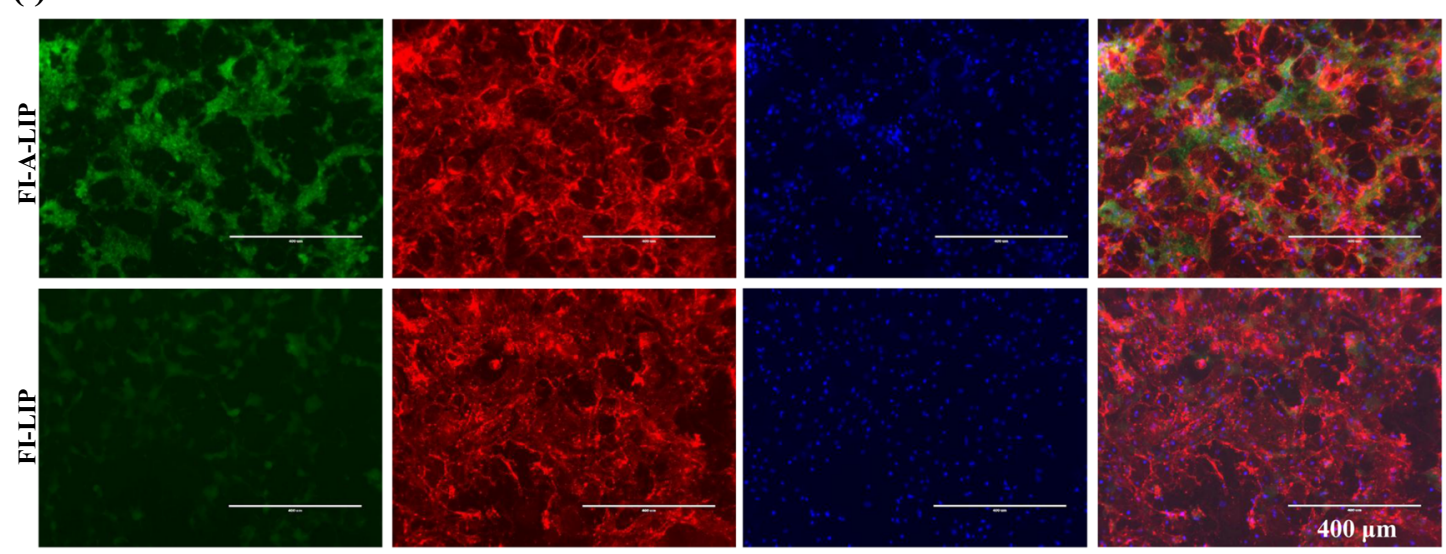

Fig. 1 Related characterization and adhesion properties of A-LIP. a The schematic formation of A-LIP. $\mathbf{b}$ The size distribution of A-LIP. $\mathbf{c}$ The size distribution of LIP. $\mathbf{d}$ The Zeta potential of A-LIP. e The Zeta potential of LIP. $\mathbf{f}$ The TEM results of A-LIP. $\mathbf{g}$ The TEM results of LIP. $\mathbf{h}$ Quantification of fluorescence of BMSCs treated with FI-A-LIP and FI-LIP labeled with Coumarin-6, which proves the adhesion of A-LIP; analysis was performed using ImageJ software. i Microscope images of BMSC monolayers treated with FI-A-LIP and FI-LIP labeled with Coumarin-6, which proves the adhesion of A-LIP. Blue: cell nuclei, stained with DAPI; Red: cell membrane, stained with CM-Dil; green: Coumarin-6-labeled liposomes. ${ }^{*} p<0.05,{ }^{* *} p<0.01,{ }^{* * *} p<$ 0.001 .

0.009, respectively, which indicates that both A-LIP and LIP had uniform sizes of particle (Fig. 1b, c). The mean potentials of A-LIP and LIP were $52.7 \pm 1.1 \mathrm{mV}$ and $-27.4 \pm 2.9 \mathrm{mV}$, respectively (Fig. 1d, e). Both A-LIP and LIP had a circular appearance (Fig. 1f, g). The introduction of octadecylamine converted the negative potential of liposomes to a positive potential, but this had little effect on the particle size of the liposome. In addition, the entrapment efficiency of these liposomes was evaluated. The entrapment efficiencies of A-LIP and LIP 
were $77.5 \pm 0.9 \%$ and $58.0 \pm 1.1 \%$, respectively. Because of the negative charge of protein and the principle of electrostatic attraction, positively charged octadecylaminemodified adhesive liposomes (A-LIP) had better encapsulation of BMP-2 than unmodified A-LIP.

The green fluorescence signal in the FI-A-LIP group was stronger than the signal in the FI-LIP group (Fig. 1i), which proved that FI-A-LIP had stronger adhesion to rat bone marrow mesenchymal stem cells (BMSCs) than LIP (Fig. 1i) ${ }^{25}$. The relative fluorescence intensities of the FIA-LIP group and FI-LIP group were $100 \pm 9 \%$ and $38 \pm 6 \%$ (Fig. 1h), respectively. Octadecylamine is a cationic material because the amino group is positively charged. Electrostatic interaction is one of the main mechanisms of nanoparticle adhesion ${ }^{33}$. For example, it has been reported that chitosan (CS) is a positively charged material, and it may form electrostatic interactions with the negative charge of the cornea and cell membrane to increase the adhesion $^{34}$. Therefore, the introduction of octadecylamine changes the potential of liposomes, which may enhance the adhesion of liposomes to cells. The cells incubated with FI-A-LIP had stronger green fluorescence (Fig. 1i), which means that more green fluorescent liposomes adhere to the cells, which proves the bioadhesive properties of FI-A-LIP.

PEG hydrogels were based on FDA-approved polyethylene glycol (PEG) homopolymer, which is extensively used in biomedical applications due to its biocompatibility and high hydrophilicity ${ }^{35}$. Specifically, commercially available SH-PEG with star-like architecture and 20,000 g $\mathrm{mol}^{-1}$ molecular weight was used in this work (Fig. S1). We crosslinked SH-PEG with $\mathrm{Ag}^{+}$by Ag-S coordination to form an antibacterial, self-healing and injectable A-LIPPEG lipo-hydrogel that was loaded with adhesive liposomes (A-LIP) to promote bone reconstruction. SEM images of lipo-hydrogels (Fig. $2 \mathrm{~d}-\mathrm{g}$ ) showed that the cross-section morphology and porosity of the lipohydrogel network were similar. Both A-LIP-PEG and LIP-PEG became typical porous microstructures in lipohydrogels after freeze drying (Fig. $2 \mathrm{~d}-\mathrm{g}$ ). The hole diameter was approximately $50-200 \mu \mathrm{m}$, which was larger than what is seen in other ordinary hydrogels, such as GelMA $^{19}$. This may be due to the relatively low crosslinking density of A-LIP-PEG and LIP-PEG. To show the performance after injection, linear viscosity measurements at alternating low and high shear rates were collected. The measurements showed that A-LIP-PEG exhibited shear-thinning behavior (Fig. S2), which resulted from the disruption of chemical cross-links between the polymer chains that occurred following the application of shear stress ${ }^{36}$. Rapid shear-thinning is ideal for injectable applications. In addition, A-LIP-PEG could be injected with a needle as small as $0.5 \mathrm{~mm}$ in diameter to produce hydrogel filaments (Fig. 2h). A-LIP-PEG had excellent injectable performance and could better fill the osteoporotic fracture area and cavity ${ }^{37}$. Implanted hydrogels after injection faced constant mechanical force, which may result in deformation or damage of the hydrogels. In this circumstance, the hydrogel with the ability to self-heal after inflicted damage will be capable of having the longest application and lifespan because the integrity of the broken hydrogel fragment after injection can be recovered at the target site under physiological conditions, which prevents a large single release of loaded bioactive molecules and improves the delivery efficiency $^{38,39}$. To examine the lipo-hydrogel's self-healing properties, the elastic modulus and viscous modulus of ALIP-PEG were validated by multicycle step strain oscillatory measurements at a constant frequency of $5 \mathrm{~Hz}$ (Fig. S3 $)^{40}$. In the first cycle, A-LIP-PEG exhibited elastic and viscous moduli of $\sim 3500 \mathrm{~Pa}$ and $\sim 850 \mathrm{~Pa}$, respectively, indicating that the elastic regime far exceeded the viscous regime at $0.5 \%$ oscillatory shear strain, suggesting that the A-LIP-PEG remained intact at low shear $\operatorname{strain}^{40}$. In contrast, the elastic modulus $(\sim 7 \mathrm{~Pa})$ was slightly lower than the viscous modulus $(\sim 16 \mathrm{~Pa})$ at $500 \%$ strain of oscillatory shear, suggesting that A-LIP-PEG exists in a liquid state during this time period. After the reversal of shear strain, the elastic regime restored its predominant over the viscous domain, indicating that the lipo-hydrogel's structure had a high recovery rate. The results significantly demonstrated the self-healing characteristics of A-LIP-PEG. Separate fragments of A-LIP-PEG that were different colors were exposed at room temperature for $30 \mathrm{~min}$, restoring A-LIP-PEG to its original state and showing self-healing characteristics (Fig. 2i). The mechanism of self-healing might be that A-LIP-PEG was crosslinked by the coordination of Ag-S and disulfide bonds (Fig. 2j) ${ }^{41}$. When the A-LIP-PEG was cut, the bonds were broken, and when they came into contact with each other, the A-LIP-PEG was restored. Self-healing properties are particularly attractive for osteoporotic fracture repair because they help reduce gel fragmentation and integration of broken gels, prolonging the service life of hydrogels.

To test the mechanical properties of lipo-hydrogels, we performed rheological analysis at different strains. The elastic modulus (4027 Pa) (G') of A-LIP-PEG decreased, and the loss modulus (1414 Pa) (G") increased with increasing strain. Finally, the elastic modulus (381.5 Pa) $(G$ ') was slightly higher than the loss modulus (37.4 Pa) (G"), while the elastic modulus (3993 Pa) (G') of LIP-PEG decreased and the loss modulus (1594 Pa) (G") increased with increasing strain. The elastic modulus (187.6 Pa) $\left(G^{\prime}\right)$ of LIP-PEG was lower than that of the loss modulus (241.4 Pa) (G") with increasing strain (Fig. 3a, b), and gelto-liquid transition occurred. This indicated that the mechanical properties of A-LIP-PEG and LIP-PEG 
(a)

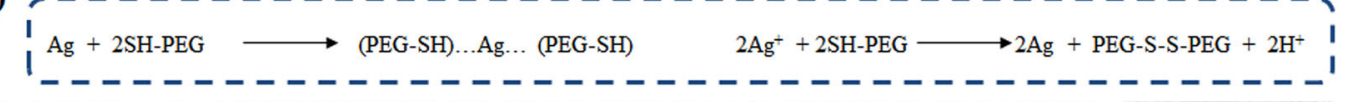

(b)
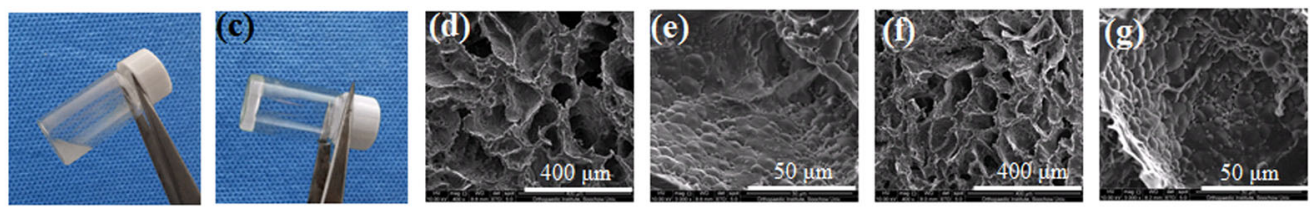

(h)
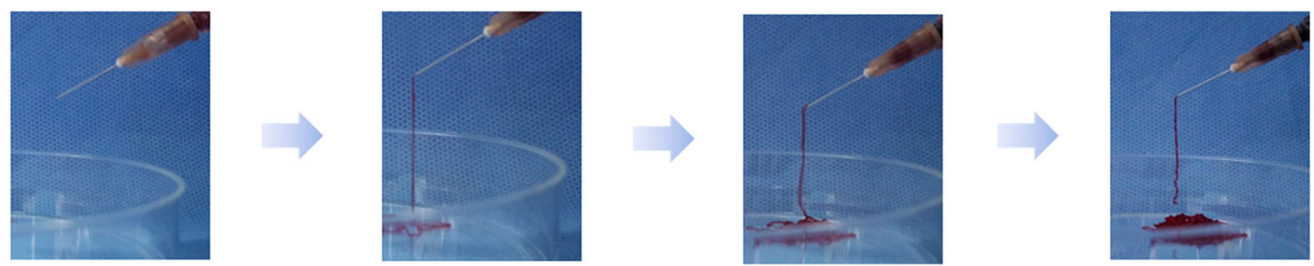

(i)
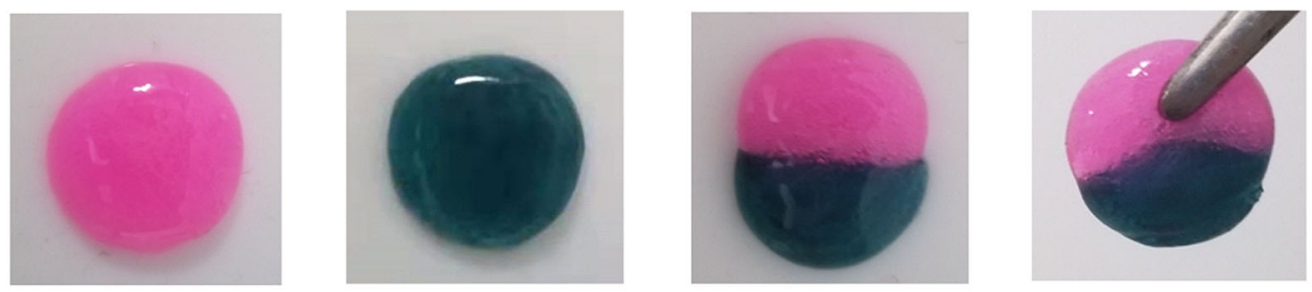

(j)
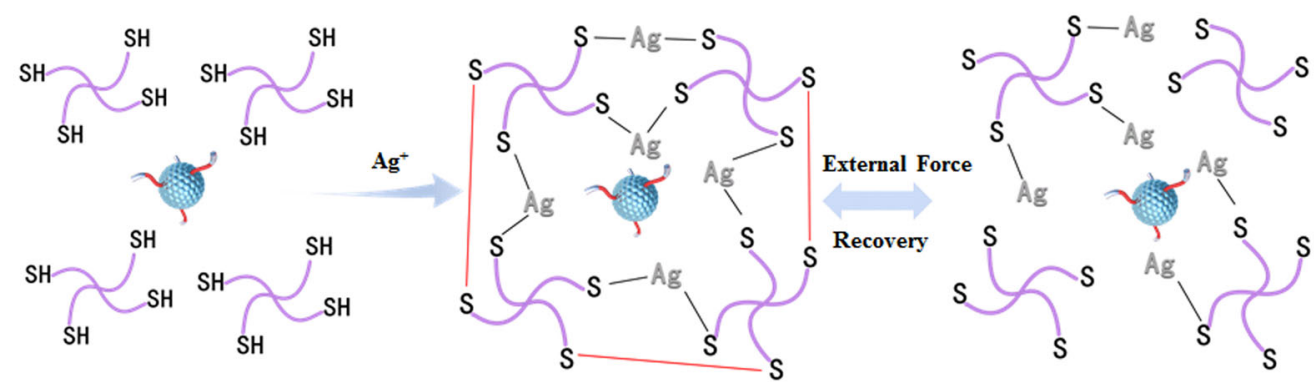

Fig. 2 Injectable and self-healing properties of A-LIP-PEG, morphological examination of A-LIP-PEG, and mechanism of A-LIP-PEG action. a Coordination and disulfide bond formation between Ag and S. b Solution of SH-PEG with A-LIP. $\mathbf{c}$ Adding Ag+ to a solution with SH-PEG and A-LIP to form the A-LIP-PEG hydrogel. $\mathbf{d}$, e The SEM results of A-LIP-PEG. $\mathbf{f}, \mathbf{g}$ The SEM results of LIP-PEG. $\mathbf{h}$ Red dye-labeled A-LIP-PEG hydrogel was injected through a 1-ml syringe with needles as small as $0.5 \mathrm{~mm}$ diameter to investigate their injectability. $\mathbf{i}$ Photographs of the self-healing performance of ALIP-PEG hydrogels. Two fragments of A-LIP-PEG hydrogels in contact for several min could heal into one unified piece. $\mathbf{j}$ The mechanism of the selfhealing ability and the injectability were both enabled by the coordination of the $\mathrm{Ag}-\mathrm{S}$ and disulfide bonds.

decreased with increasing strain, which illustrated the shear thinning and injectable properties of the lipohydrogels.

Figure 3c shows the release profile of BMP-2 from ALIP-PEG and LIP-PEG. On the first day, the cumulative release of A-LIP-PEG was $41.1 \pm 0.6 \%$, which was similar to that of LIP-PEG $(44.8 \pm 6.9 \%)$. At the end of the experiment, the cumulative release of A-LIP-PEG was $60.3 \pm 2.1 \%$, which was lower than that of LIP-PEG $(64.3 \pm 9.0 \%)$. The release time of BMP-2 for both ALIP-PEG and LIP-PEG was $9 \mathrm{~d}$, indicating that the drug release performance of A-LIP-PEG was similar to that of LIP-PEG. This may be because the introduction of octadecylamine had no effect on the density of the cross- linking network of lipo-hydrogel. Specifically, these drugs would be released in several different ways in vitro. On the one hand, these drugs would be released from the cavum with the leakage of liposomes. On the other hand, these drug-loaded liposomes would be released from the composite hydrogel, and then these drugs could be detected in the released medium. To explore the mechanism used in lipo-hydrogel formation, we collected Fourier transform infrared spectroscopy (FTIR) spectra of A-LIP-PEG. The FTIR spectra collected from the A-LIP-PEG samples had vibration signals from the disulfide bonds $(v(\mathrm{~S}-\mathrm{S})$, $530 \mathrm{~cm}^{-1}$ ) (Fig. 3d) ${ }^{41}$. SH was prone to forming disulfide bonds, resulting in hydrogel formation. The coordination between $\mathrm{Ag}$ and $\mathrm{S}$ and the disulfide bond were two ways 

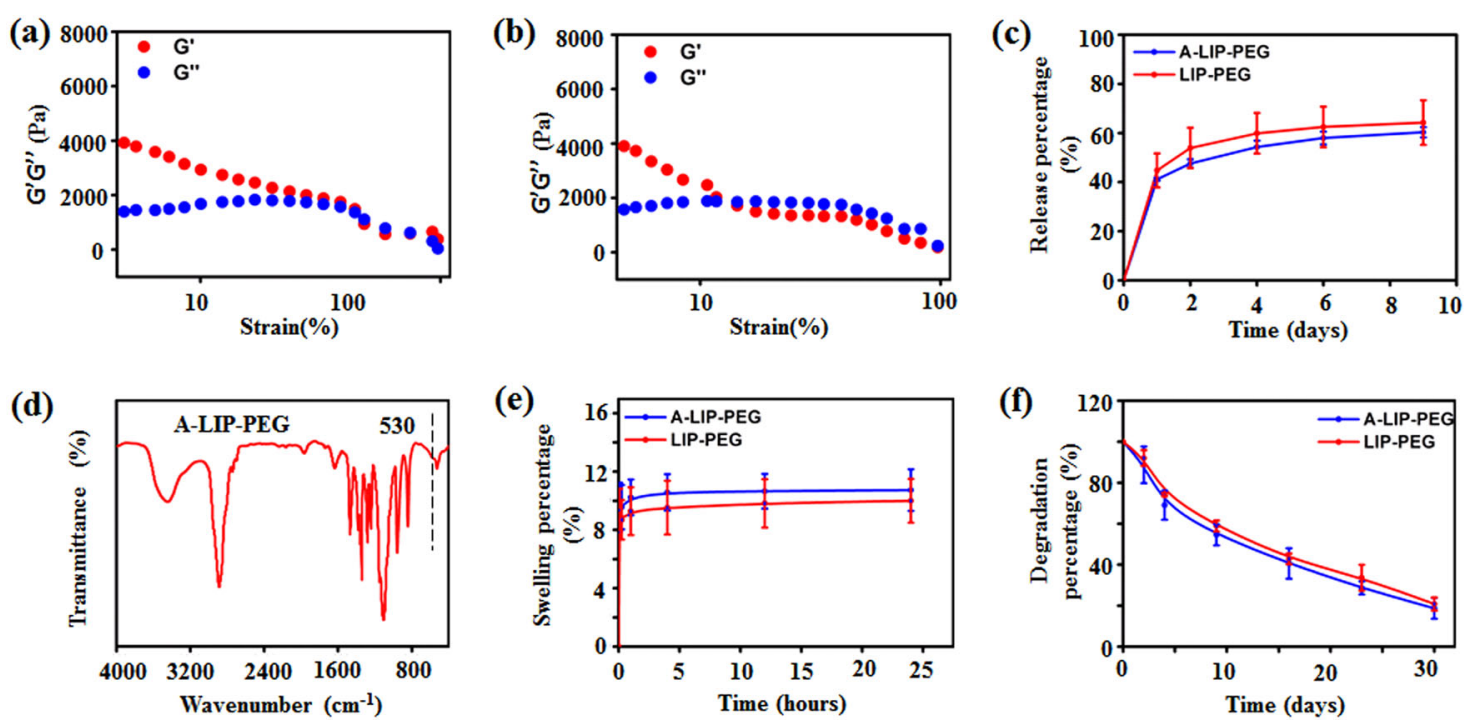

Fig. 3 Rheology, release, FTIR, swelling test, and weight loss analysis of lipo-hydrogels. a The storage modulus $G^{\prime}$ and the loss modulus $G^{\prime \prime}$ of A-LIP-PEG were plotted logarithmically against different strains. $\mathbf{b}$ The storage modulus $G^{\prime}$ and loss modulus $G^{\prime \prime}$ of LIP-PEG were plotted

logarithmically against different strains. The frequency was $10 \mathrm{~Hz}$ with a dynamic strain scanning from 4 to $100 \%$. c In vitro BMP-2 release measured in PBS. $\mathbf{d}$ Fourier transform IR spectra of A-LIP-PEG hydrogel. e Swelling percentage of lipo-hydrogels in PBS. f Degradation of lipo-hydrogels in PBS.

that the lipo-hydrogel formed ${ }^{41}$. A-LIP-PEG reached swelling equilibrium at $24 \mathrm{~h}$, and the swelling ratio reached $1070 \pm 140 \%$; LIP-PEG reached swelling equilibrium at $24 \mathrm{~h}$, and the swelling ratio reached $1000 \pm 150 \%$ (Fig. 3e). These results indicate that both lipo-hydrogels had similar amounts of water penetration and volume change $^{19}$. The swelling ratio reflected the density of the internal network of the lipo-hydrogel. The smaller the equilibrium swelling rate was, the closer the lipo-hydrogel network was. The swelling ratio of A-LIP-PEG was almost the same as that of LIP-PEG, which suggested that the density of the internal network of the two lipo-hydrogels was basically the same, and octadecylamine had no effect on the density of the cross-linking network. Dense hydrogel networks could act as an obstacle to the movement of water molecules ${ }^{42}$. The mass loss of lipohydrogels could also reflect their stability. After soaking lipo-hydrogels in PBS for $28 \mathrm{~d}$, the weight loss rate of ALIP-PEG was similar to that of LIP-PEG, with rates of $81.3 \pm 5.1 \%$ and $79.2 \pm 3.2 \%$ (Fig. 3f), respectively. The long degradation time of LIP-PEG and A-LIP-PEG might be attributed to stable bonding, which would not be easily broken by water penetration. From these results, we infer that A-LIP-PEG had good structural stability and was beneficial for the sustained release of drugs, which is very important for the repair of osteoporotic fractures, since osteoporotic fracture healing takes longer than the healing of other tissues.

The interaction between liposomes and BMSCs was qualitatively studied by fluorescence microscopy. There was no green fluorescence signal on the 6-well plate, which proved that there was no interaction between the cell and the FI-LIP released from FI-LIP-PEG (Fig. 4a). However, green fluorescence signals could be observed in the 6-well plate with FI-A-LIP-PEG. The relative fluorescence intensities of the FI-A-LIP-PEG group and FILIP-PEG group were $100 \pm 18 \%$ and $33 \pm 6 \%$ (Fig. S4), respectively. This was due to the adhesion of FI-A-LIP released from FI-A-LIP-PEG, which reduced the possibility of FI-A-LIP being washed away by PBS $^{25}$. According to this result, FI-A-LIP could be released from FI-A-LIPPEG and could still show significant adhesion. After i.p. injection of DIR-A-LIP, the fluorescent signal was observed for up to 3 days (Fig. S5). In contrast, after i.p. injection of DIR-LIP, there were no fluorescent signals. We drew ROIs on each individual mouse, and the average radiance is presented in Fig. S6. The results showed that DIR-A-LIP had obvious adhesion to tissues in vivo and could prolong the local retention time of liposomes and improve the bioavailability of liposomes. At present, there are few studies on the combination of adhesive liposomes in hydrogels because traditional adhesive liposomes do not maintain adhesion properties well after release from hydrogels. For example, the adhesion of liposomes with chitosan derivatives has been widely known ${ }^{43}$. However, chitosan derivatives coat the surface of liposomes and were not directly connected to liposomes, which resulted in weak connections between chitosan derivatives and liposomes. Chitosan derivatives of adhesive liposomes dissociated from hydrogel were easily dissolved and diluted by body fluids, resulting in the loss of adhesion by liposomes. However, octadecylamine could be carried in 


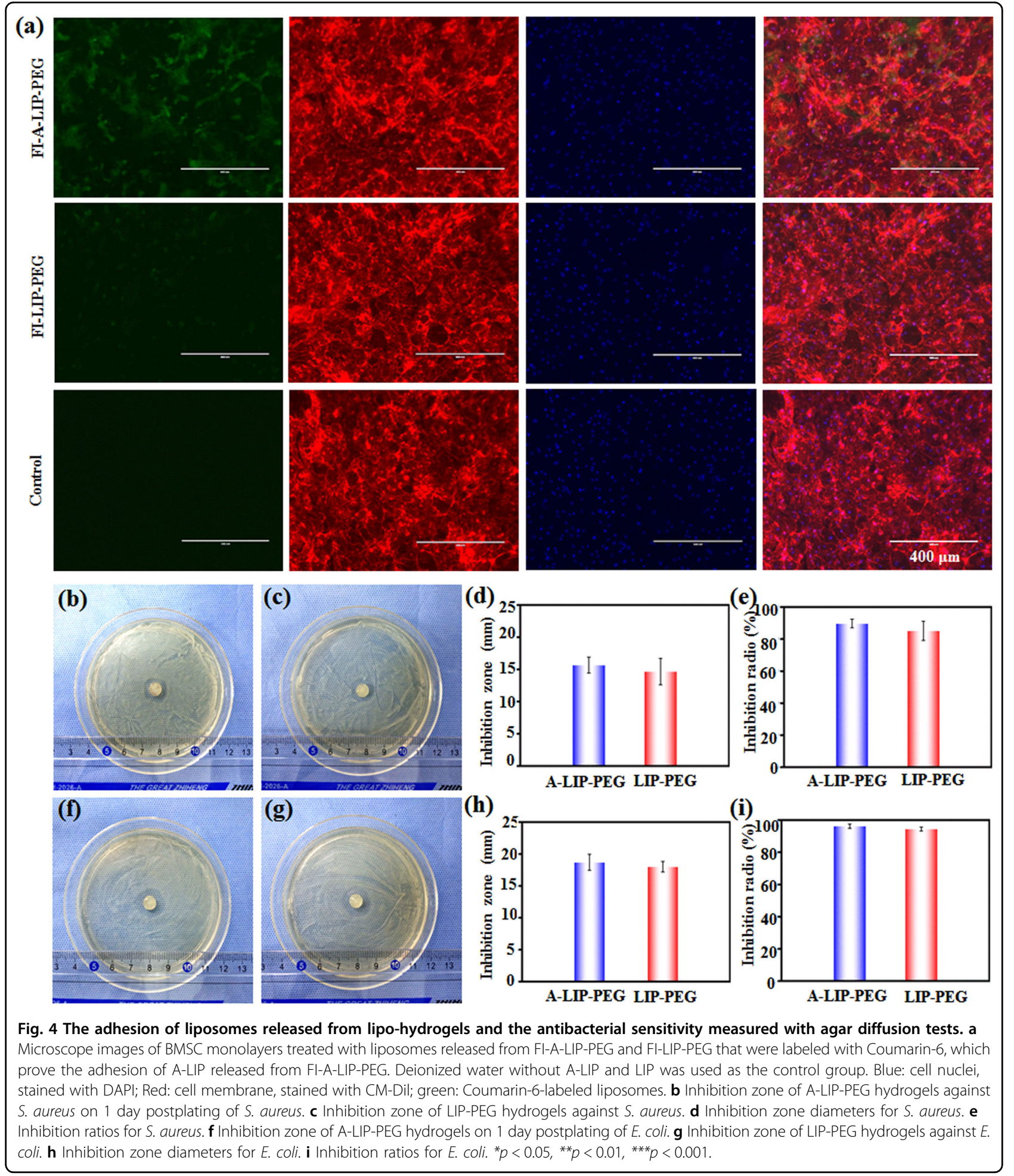

the phospholipid bilayer rather than coating the outside of the liposomes because of the strong hydrophobicity of octadecylamine $^{44}$. In this way, when liposomes dissociate from hydrogel, octadecylamine would not be diluted or washed away by bodily fluid, which might be the reason why A-LIP in A-LIP-PEG maintain adhesion properties. This performance was helpful for maintaining the drug concentration, inhibiting the dilution of the drug in the body fluids and allowing targeting and localization of a drug to a specific site ${ }^{45}$. 
To study an appropriate clinical treatment model of infection, S. aureus and Escherichia coli were adopted as representative Gram-positive and Gram-negative bacteria, respectively, and they were used to evaluate the antibacterial ability of our lipo-hydrogels ${ }^{41}$. At present, after clinical fracture surgery, there was still a potential risk of bacterial infection, such as improper operation procedures by medical staff and improper activity of patients. If the wound infection was mild, the wound healing time would be prolonged, and in severe cases, the fracture would not be repaired. At present, to address potential infections, antibiotics are still used in the clinic to resist infection. On the one hand, with whole-body antibiotic treatment of local infections, the drug utilization rate is low; on the other hand, antibiotics can easily cause bacteria to develop drug resistance ${ }^{46}$. In this work, $\mathrm{Ag}^{+}$was used not only as a cross-linking agent of A-LIP-PEG but also as an antibacterial agent. $\mathrm{Ag}^{+}$has many advantages in dealing with potential infections, such as having a wide antibacterial spectrum, strong antibacterial ability and no drug resistance ${ }^{47}$. The bacterial inhibition halos around the A-LIP-PEG were $\sim 15.7 \pm 1.2 \mathrm{~mm}$ for $S$. aureus (Fig. $4 \mathrm{~b}$ ) and $18.7 \pm 1.2 \mathrm{~mm}$ for E. coli (Fig. $4 \mathrm{f}$ ), and those around LIP-PEG were $\sim 14.7 \pm 2.1 \mathrm{~mm}$ for $S$. aureus (Fig. 4c) and $18.0 \pm 0.8 \mathrm{~mm}$ for E. coli (Fig. 4g). The bacterial viability was also evaluated using a live/dead (green/red) staining assay. Figure $\mathrm{S} 7$ shows fluorescent images of $S$. aureus and E. coli. Red fluorescence was observed only in the A-LIP-PEG and LIP-PEG groups, i.e., the bacteria were dead, indicating the great antibacterial ability of the treatment; green fluorescence was observed in the control group, i.e., the bacteria were alive. To quantify the antibacterial activity and assess bacterial inhibition by the lipo-hydrogels, the number of colonies was counted. The bacterial inhibition rates of A-LIP-PEG and LIP-PEG against $S$. aureus were $89.7 \pm 2.3 \%$ and $85.1 \pm 6.0 \%$, respectively, indicating that A-LIP-PEG had strong antibacterial properties (Fig. 4e). For E. coli, the bacterial inhibition rates of A-LIP-PEG and LIP-PEG were $96.2 \pm$ $1.4 \%$ and $94.5 \pm 1.2 \%$ (Fig. 4i), respectively. In this work, the mechanism of antibacterial activity of A-LIP-PEG might be attributed to the release of $\mathrm{Ag}^{+}$from A-LIPPEG; following its release, $\mathrm{Ag}^{+}$would display superior antibacterial activity by interfering with the membrane permeability, inhibiting respiratory enzymes and condensing $\mathrm{DNA}^{48}$. These results suggest that A-LIP-PEG had broad-spectrum antibacterial activity against Grampositive and Gram-negative bacteria, which hopefully reduce the potential risk of infection after osteoporotic fracture surgery.

As a biomaterial for osteoporotic fracture healing, the biocompatibility of A-LIP-PEG is very important. Therefore, we used CCK- 8 to quantitatively investigate the proliferation of BMSCs. As shown in Fig. S5, compared with the control group, slightly decreased cell viability was observed for cells after incubation for 7 days with A-LIPPEG and LIP-PEGd. However, there was no significant difference in the optical density (OD) value among the groups, and the optical density (OD) value increased with time (Fig. S8). As an antibacterial agent, Ag had low biological toxicity ${ }^{47}$. A team of researchers reported a matrix sterilization hydrogel coating based on Ag nanoparticles. When the loading amount of $\mathrm{Ag}^{+}$in the coating was less than $9.0 \mu \mathrm{g} / \mathrm{cm}^{2}$, the membrane showed no cytotoxicity ${ }^{49}$. In our study, the A-LIP-PEG hydrogel was only loaded with $0.81 \mu \mathrm{g} \mathrm{Ag}^{+}$, which was much lower than the cytotoxicity threshold. Moreover, $\mathrm{Ag}^{+}$is used not only as an antimicrobial agent in the hydrogel but also as a cross-linking agent of the hydrogel. Compared with the direct loading of $\mathrm{Ag}^{+}$in hydrogels, the Ag in A-LIP-PEG reported in this paper bound to SH-PEG, resulting in the slower release of $\mathrm{Ag}^{+}$, which reduced the biological toxicity of $\mathrm{Ag}^{+}$. In this paper, $\mathrm{Ag}$ was used as a crosslinking agent to bond with SH-PEG to form Ag-SH. With the slow degradation of A-LIP-PEG in vivo, the bonds were gradually broken, and the $\mathrm{Ag}^{+}$was slowly released, which reduced the biotoxicity of $\mathrm{Ag}^{+}$. This might be the reason for the good biocompatibility of A-LIP-PEG. In addition, as a biodegradable organic compound, octadecylamine had low biological toxicity ${ }^{50}$. There were no adverse effects observed for two years in mice fed with $500 \mathrm{mg} / \mathrm{L}$ octadecylamine ${ }^{51}$. It was reported that a new lipoprotein-mimic nanocarrier was developed by a research team, which was composed of a hydrophobic core and surrounded by an amphiphilic phosphatidylcholine (PC) shell ${ }^{52}$. The hydrophobic core was composed of medium chain triglyceride and octadecylamine and stabilized by the PC layer. In the biocompatibility test, the material had no toxic effect on U20P cells, HepG2 cells and Bel 7402 cells, which proved that octadecylamine has good biocompatibility. In addition, the live/dead results from BMSCs were used to assess the effect of ALIP-PEG on cell viability (Fig. 5a). There was no significant difference among the A-LIP-PEG, LIP-PEG and control groups, which further proved the good biocompatibility of A-LIP-PEG. The morphology of the cells seeded on the lipo-hydrogels was very good (Fig. 5b). The adhesion effect of cells to the A-LIP-PEG hydrogel was slightly worse than it was in the 24-well plate because the PEG hydrogel had a certain anti-fouling effect and reduced the adhesion of cells to hydrogel. However, the A-LIP-PEG hydrogel still exhibited adhesion to cells and can be used as an excellent biomaterial. The results showed that A-LIP-PEG is biocompatible with BMSCs and a biocompatible bone regeneration material.

To investigate the ability of A-LIP-PEG and LIP-PEG to promote early stages of osteogenesis, the osteogenic differentiation ability of A-LIP-PEG and LIP-PEG was 


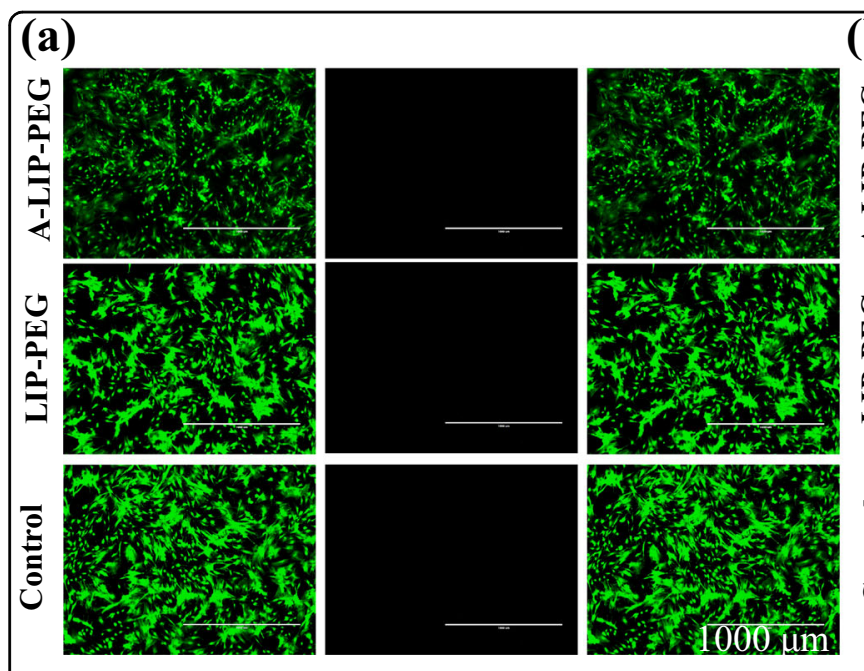

(b)

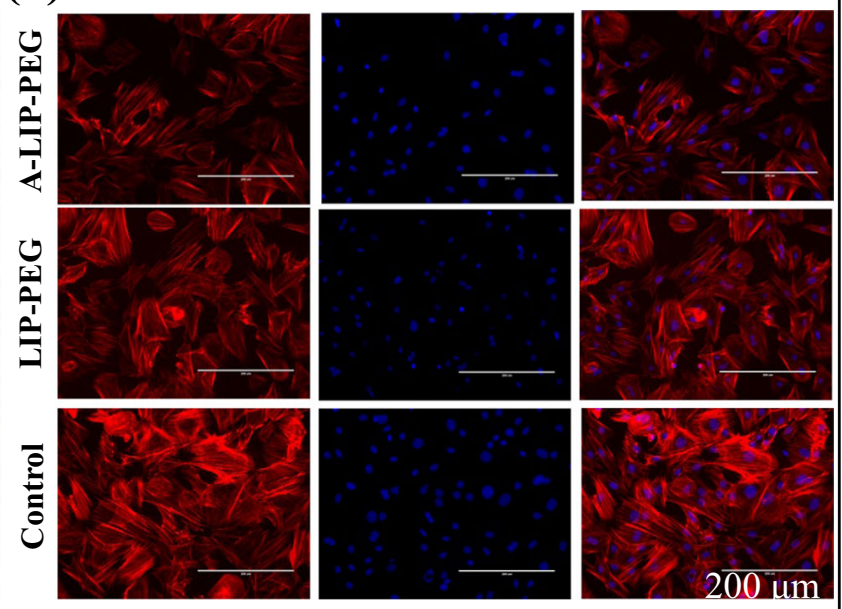

Fig. 5 The biocompatibility of lipo-hydrogels. a Live/dead staining of cells cultured in extracted release buffer from different hydrogels for 2 days. b Images of the BMSCs cultured on the hydrogel after 1 day; cells are stained with phalloidin (red) and DAPI (blue).

evaluated by ALP activity. Although the loading of BMP-2 was the same, ALP staining 7 days later showed that the ALP expression in BMSCs cultured with A-LIP-PEG was significantly higher than that of the LIP-PEG culture group or the control group (Fig. 6a). The in vitro osteogenic study confirmed the adhesion of A-LIP released by A-LIP-PEG to improve the bioavailability of BMP-2. In addition, the osteogenic ability of A-LIP-PEG and LIPPEG in the long term was evaluated by the observation of calcium nodules. The levels of calcium nodules after 14 and 21 days of culture in the A-LIP-PEG group were higher than they were in the LIP-PEG and control groups (Fig. 6a), which showed that the osteogenic differentiation of A-LIP-PEG cultured cells was higher than that of LIPPEG cultured cells. These results suggested that A-LIPPEG is an ideal osteogenic differentiation material.

The expression of osteocalcin (green) was stronger in the cells cultured with A-LIP-PEG than it was in other groups, which indicated that the A-LIP released by A-LIPPEG enhanced the osteogenic differentiation of BMSCs (Fig. 6b). However, the signal expressed in the cells cultured with LIP-PEG was slightly weaker, and the signal in the control group was very weak. The relative fluorescence intensities of the A-LIP-PEG group, LIP-PEG group and control group were $100 \pm 20 \%, 46 \pm 8 \%$, and $4 \pm 1 \%$ (Fig. 6c), respectively, which might be because LIP released by LIP-PEG could not adhere to the cells, resulting in the rapid loss of BMP-2. Therefore, it was difficult to achieve the concentration of drugs needed to maintain osteogenic activity ${ }^{53}$. As shown in Figs. S9-11, osteopontin (OPN), osterix (OSX), and type 1 collagen (COL 1) were investigated by real-time quantitative polymerase chain reaction (RT-QPCR) analysis. The expression of OPN, OSX, and COL 1 in the A-LIP-PEG samples was obviously higher than those of the other groups at $5 \mathrm{~d}$. The results showed that A-LIP-PEG promoted the expression of OPN, OSX, and COL 1 compared to LIP-PEG and the control.

To confirm the successful establishment of the osteoporosis model, the osteoporotic fracture healing of ovariectomized (Ovx group) and sham operated rats (Sham group) was studied by micro-CT analysis 12 weeks after ovariectomy. The femurs in the Ovx group showed reduced trabecular bone and disordered trabecular structure and enlarged medullary cavity compared with those of the Sham group (Fig. 7a, b). Micro-CT quantitative analysis further revealed that the bone mineral density (BMD), bone volume fraction (BV/TV) and the number of bone trabeculae ( $\mathrm{Tb} . \mathrm{N}$ ) in the Ovx group were $38 \%, 52$ and $42 \%$ lower than they were in the Sham group (Fig. 7c, d, g), respectively. The trabecular separation (Tb. Sp) increased by $50 \%$ compared with the Sham group (Fig. $7 \mathrm{f})$. These results confirmed that the osteoporosis model had been well established in rats.

$\mathrm{X}$-ray morphogenesis of bone at the fracture site was observed in the process of fracture healing (Fig. 7e) ${ }^{31}$. After 2 weeks of treatment, the fracture line was slightly blurred, and callus growth occurred in the A-LIP-PEG treatment group; however, the fracture line was still clear in the LIP-PEG group and the control group, and no callus was found. After 4 weeks of treatment, the fracture line of the A-LIP-PEG group basically disappeared, and a callus filled the defect; further, a callus appeared in the LIP-PEG group, and no callus was observed in the control group. After 8 weeks of treatment, the callus and cortical density of the A-LIP-PEG group were the same, and they connected with each other, indicating fracture healing. At the same time point, the amount of callus increased in the 


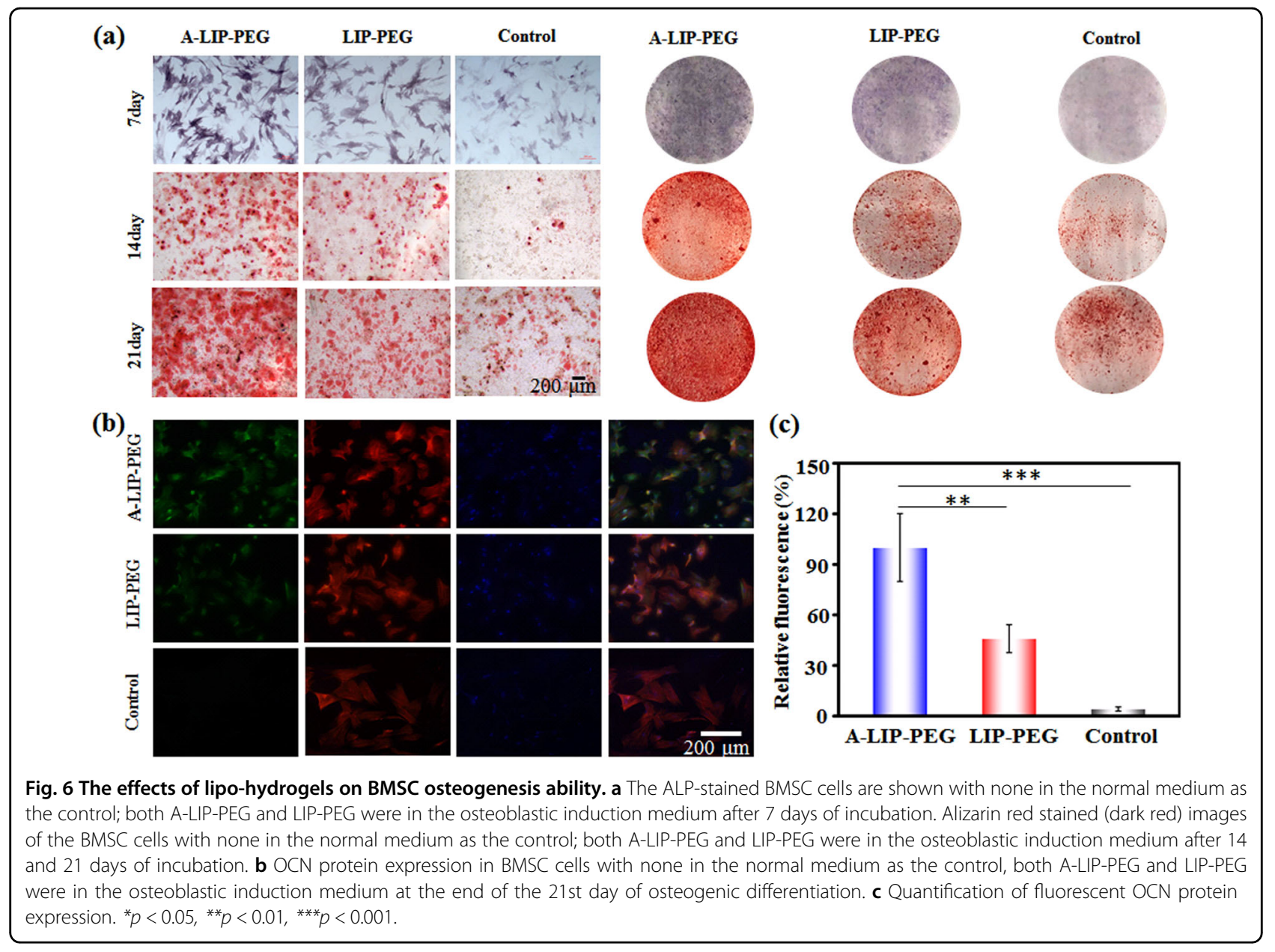

LIP-PEG group, while the fracture line in the control group was blurred, and callus appeared. The results showed that A-LIP-PEG is an ideal osteogenic material, which might be because the degradation and release of ALIP-PEG in vivo produced adhesion to the tissue around the fracture, prolonging the retention time of liposomes and maintaining the drug concentration needed for local fracture repair for a sustained period. To further evaluate bone healing, micro-CT was used to assess bone formation after treatment. After 8 weeks of treatment, the healing tissue around the fracture in the A-LIP-PEG treatment group showed greater mineralization than it did in the LIP-PEG treatment group and the control group (Fig. $7 \mathrm{~h}, \mathrm{k}$ ). The quantitative micro-CT data were consistent with the observed results. Compared with LIP-PEG BV $\left(90.7 \pm 4.2 \mathrm{~mm}^{3}\right)$, the BV in the A-LIP-PEG group $\left(178.6 \pm 16.5 \mathrm{~mm}^{3}\right)$ increased significantly, indicating a significant improvement in bone mineralization (Fig. 7i). Quantitatively, the bone volume fraction (BV/TV) of the A-LIP-PEG group $(86 \pm 3.6 \%)$ was significantly higher than it was in the LIP-PEG group $(69 \pm 2.7 \%)$ and control group $(56 \pm 0.4 \%)$ at the 8 th week (Fig. $7 j)$. The results showed that bone mineralization at the fracture was significantly improved. Similarly, the trabecular microstructure of the A-LIP-PEG group showed the best bone condition. Compared with LIP-PEG $(0.609 \pm 0.121 \mathrm{~mm})$ and the control group $(0.526 \pm 0.023 \mathrm{~mm})$, the trabecular thickness $(\mathrm{Tb}$. Th) of a femur treated with A-LIP-PEG $(0.907 \pm 0.051 \mathrm{~mm})$ also increased by 1.49 -fold and 1.72 fold, respectively (Fig. $7 \mathrm{~m}$ ). In addition, the trabecular separation (Tb. Sp) of a femur treated with A-LIP-PEG $(0.112 \pm 0.026 \mathrm{~mm})$ was significantly lower than it was following treatment with LIP-PEG $(0.310 \pm 0.048 \mathrm{~mm})$ and in the control group $(0.878 \pm 0.090 \mathrm{~mm})$ (Fig. 7l). These results suggested that A-LIP-PEG could accelerate local osteogenesis, which might be due to the excellent cell adhesion and bone formation promotion qualities of A-LIP-PEG. Histological examination was used as a direct method to study the bone morphology around fractures. Histological analysis of the callus showed that compared with the control group, the fracture healing in the A-LIPPEG treatment group was enhanced, which was consistent with the micro-CT quantitative analysis. HE staining also showed that compared with LIP-PEG and the control 

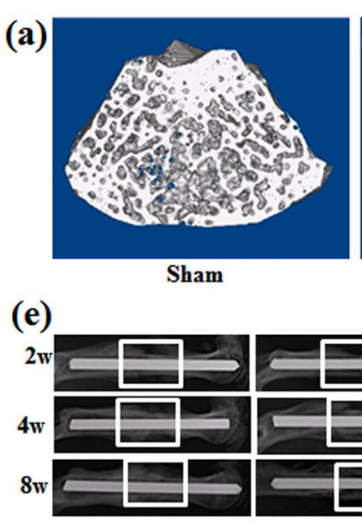

A-LIP-PEG

(h)
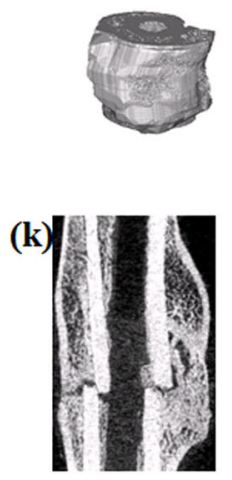

A-LIP-PEG
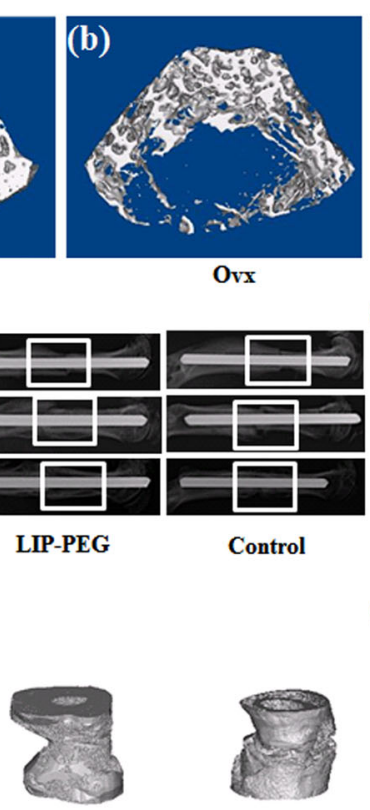

Ovx

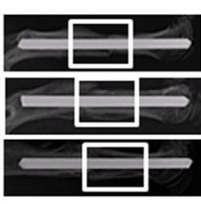

LIP-PEG

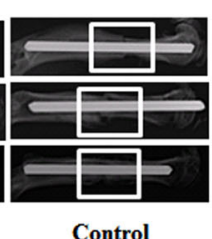

Control
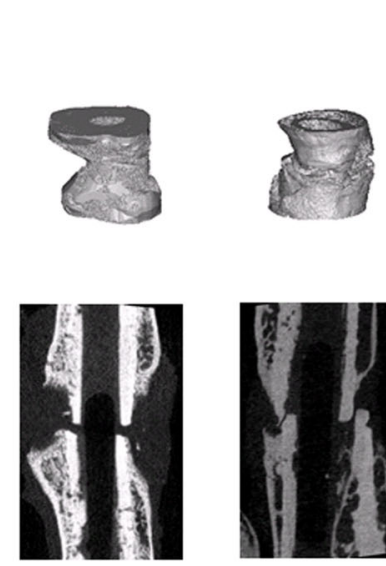

LIP-PEG

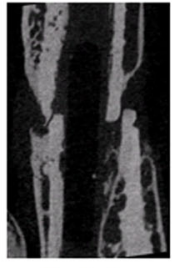

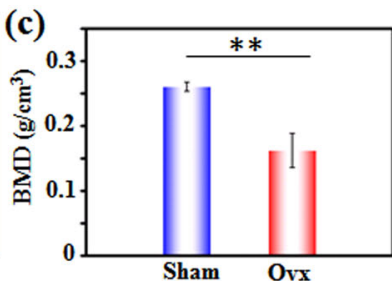
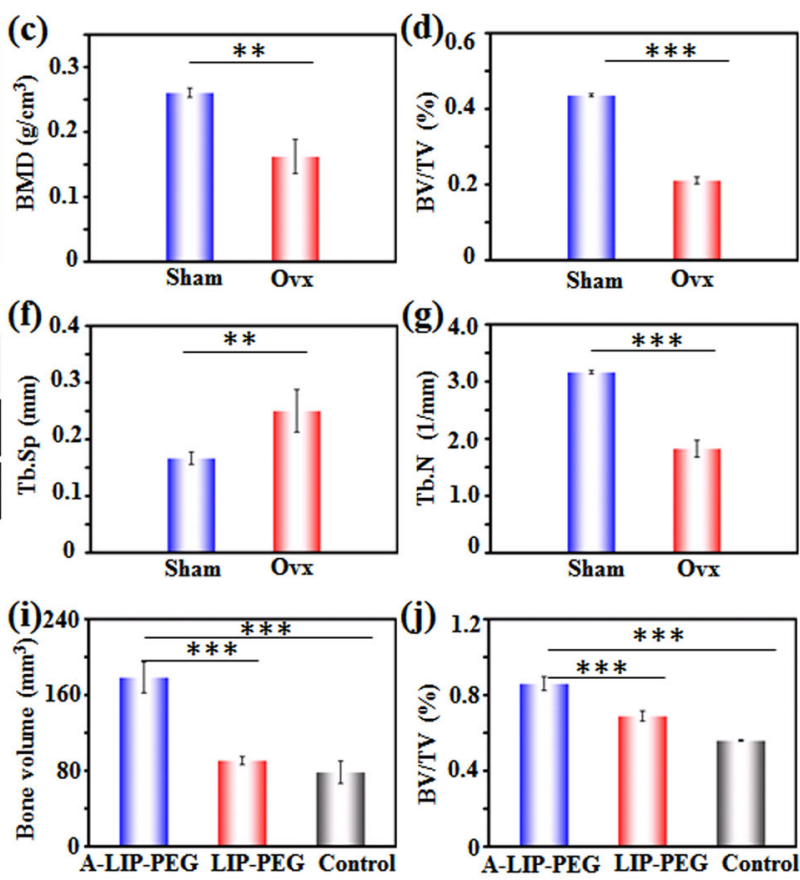

(m)
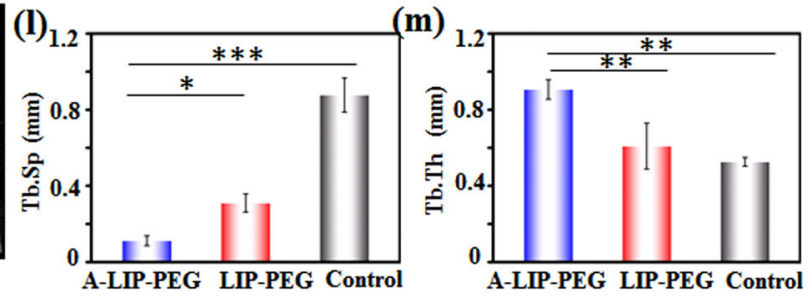

Fig. 7 Identification of femoral osteoporosis and osteoporotic fracture healing efficiency, as determined by radiographs and micro-CT analysis. $\mathbf{a}$, b Representative micro-CT images of femoral condyles in the Sham and Ovx groups confirmed the successful establishment of an osteoporosis model. $\mathbf{c}, \mathbf{d}, \mathbf{f}, \mathbf{g}$ Quantitative results of the bone morphological alterations, including bone mineral density (BMD), bone volume to tissue volume (BV/TV), trabecular space (Tb. Sp) and numbers of trabecular bone (Tb. N) in the region of interest. e Radiographs taken at 2, 4, and 8 weeks showed callus formation and fracture healing. $\mathbf{h}$ 3D reconstructed images of mineralized bone formation in the fracture area after 8 weeks of treatment. $\mathbf{k}$ Micro-CT scans were taken 8 weeks posttreatment to assess in vivo bone callus formation. $\mathbf{i}, \mathbf{j}, \mathbf{I}, \mathbf{m}$ Quantitative results of bone morphology alterations, including bone volume (BV), bone volume to tissue volume (BV/TV), trabecular space (Tb. Sp) and trabecular thickness (Tb. Th) in the region of interest. ${ }^{*} p<0.05,{ }^{* *} p<0.01,{ }^{* * *} p<0.001$.

group, the A-LIP-PEG group had less fibrous connective tissue around the fracture, more new bone content and increased bone tissue maturity (Fig. 8a). To further demonstrate the healing effect of the fracture, safranin $\mathrm{O}$ and fast green staining were performed 8 weeks after implantation for bone histopathology analysis. The ALIP-PEG group showed more mature bone tissue (dyed green) and less cartilage (dyed red) than the LIP-PEG and control groups (Fig. 8b). Masson staining showed that callus sections from the control group were mainly surrounded by cartilage and collagen fibers (dyed blue), which is consistent with the relatively early stage of fracture repair (Fig. 8c). In contrast, in the callus sections treated with A-LIP-PEG, the fracture site had been replaced by highly mineralized bone tissue (dyed red), showing early endochondral ossification. The bone area in the A-LIP-PEG group was $69.9 \pm 4.4 \%$, while that in the LIP-PEG group and the control group was only $47.3 \pm$ $0.2 \%$ and $42.2 \pm 6.8 \%$, respectively (Fig. $8 \mathrm{~d}$ ). In contrast, the cartilage area of the A-LIP-PEG group was $14.5 \pm$ 9.3\%, while that of the LIP-PEG group and the control group was $35.8 \pm 11.4 \%$ and $35.1 \pm 7.9 \%$, respectively (Fig. $8 \mathrm{e})$. The callus area in the A-LIP-PEG group $(8.9 \pm 0.5$ $\left.\mathrm{mm}^{2}\right)$ was larger than it was in the LIP-PEG group (6.4 \pm $\left.2.0 \mathrm{~mm}^{2}\right)$ and the control group $\left(5.8 \pm 0.7 \mathrm{~mm}^{2}\right)$ (Fig. 8f). It was not difficult to conclude that A-LIP-PEG hydrogeltreated fractures showed early endochondral ossification, accelerated mineralization, and reduced fibrotic tissue formation. For the difference between the A-LIP-PEG and LIP-PEG groups, A-LIP released by A-LIP-PEG might adhere to tissue, enabling a sustained release. Such sustained release could maintain an effective drug 
(a) (b)

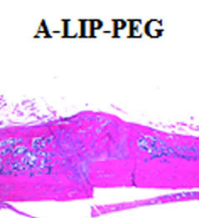

LIP-PEG
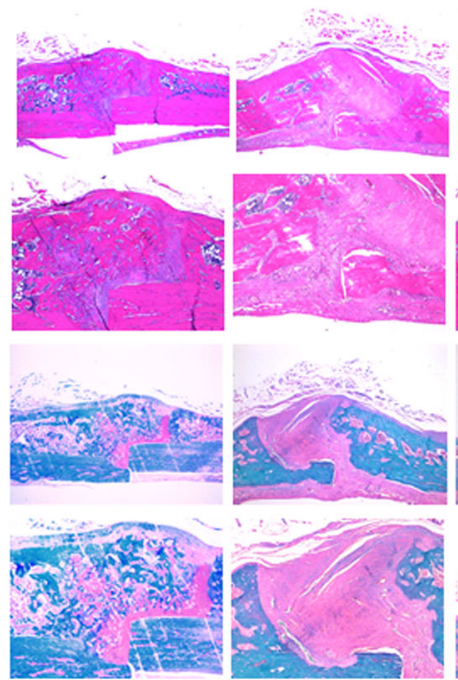

(c)
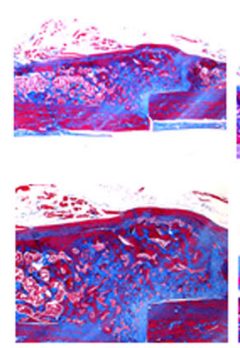
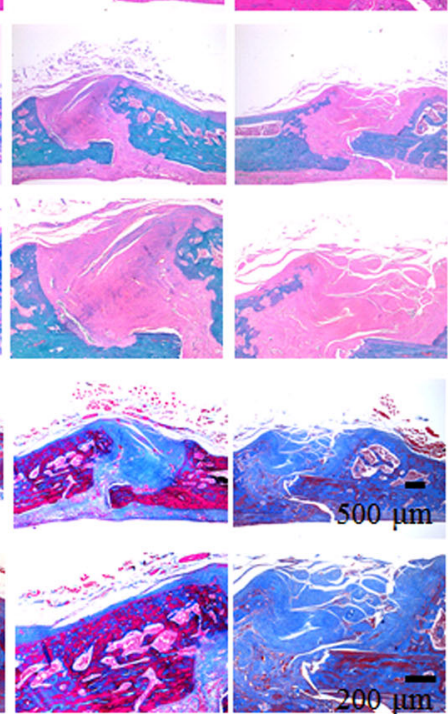

Control
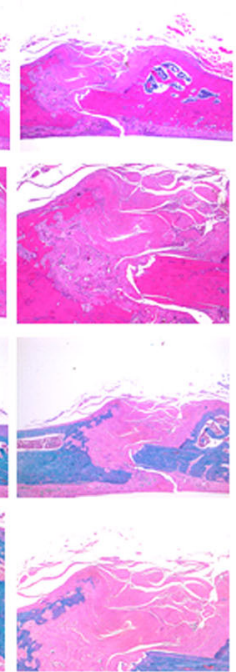

(f)

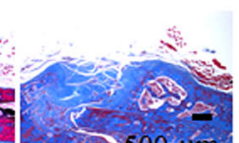

(e) (d)
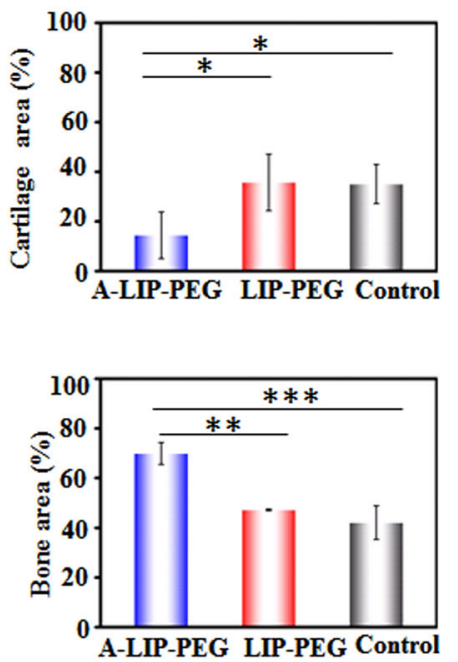

(f)

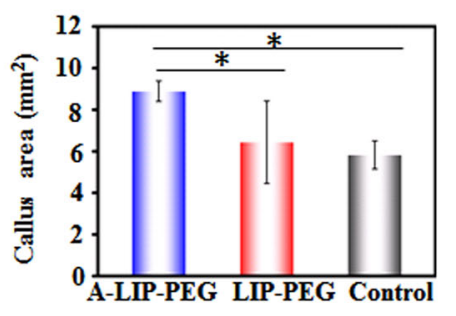

Fig. 8 Osteoporotic fracture healing studies of SD rats treated with lipo-hydrogels in vivo. a HE staining of osteoporotic fracture areas for 8 weeks. $\mathbf{b}$ Safranin $\mathrm{O}$ and fast green staining of osteoporotic fracture areas. $\mathbf{c}$ Masson's trichrome staining of osteoporotic fracture areas. The area of d bone, e cartilage, and $\mathbf{f}$ callus were quantified using ImageJ software. ${ }^{*} p<0.05,{ }^{* *} p<0.01,{ }^{* * *} p<0.001$.

concentration for a longer period of time, which would be helpful for improving bone tissue regeneration.

\section{Conclusions}

In this paper, based on the coordination cross-linking principle of $\mathrm{SH}-\mathrm{Ag}$, we developed an injectable, antibacterial, and self-healing A-LIP-PEG with A-LIP. A-LIPPEG was injected directly into osteoporotic fracture sites as well as the adjacent bone marrow cavity, and bone reconstruction was performed in situ by local release of adhesive liposomes. The A-LIP released from the A-LIPPEG maintained adhesion properties and continued to adhere to the tissue around the fracture, thus giving full play to the dual effect of the hydrogel and the drug. In addition, in vitro, A-LIP-PEG showed excellent injectable, antibacterial, and self-healing properties, which reduces the risk of postoperative infection and requires only a minimally invasive injection into the cavity. Furthermore, in vivo experiments showed that compared with LIP-PEG, A-LIP-PEG was better at promoting osteogenic differentiation. Therefore, the hydrogel drug loading system provided a new treatment method for cavity diseases. Our
A-LIP-PEG could be useful in several clinical circumstances, such as inhibition of ipsilateral recurrence in adjuvant therapy for the focal treatment of cancer, diabetic skin wound regeneration and therapies for orthopedic implant infections.

\section{Acknowledgements}

This work was supported by the National Natural Science Foundation of China (51873107 and 31870959), the Science and Technology Commission of Shanghai Municipality (18ZR1434200), the Shanghai Municipal Health and Family Planning Commission (201840027), the Shanghai Municipal Education Commission Gaofeng Clinical Medicine Grant Support (20171906), the Shanghai Jiao Tong University "Medical and Research" Program (ZH2018ZDA04), and the Shanghai talent development fund (2018099).

\section{Author details}

${ }^{1}$ Shanghai Key Laboratory for Prevention and Treatment of Bone and Joint Diseases, Shanghai Institute of Traumatology and Orthopaedics, Ruijin Hospital, Shanghai Jiao Tong University School of Medicine, 197 Ruijin 2nd Road, Shanghai 200025, P. R. China. ${ }^{2}$ Orthopedic Institute, Soochow University, 708 Renmin Rd., 215006 Suzhou, Jiangsu, China. IInternational Department, Hangzhou High School, 1958 Zhijiangdong Road, Jianggan District, 310021 Hangzhou, Zhejiang, P. R. China

Conflict of interest

The authors declare that they have no conflict of interest. 


\section{Publisher's note}

Springer Nature remains neutral with regard to jurisdictional claims in published maps and institutional affiliations.

Supplementary information is available for this paper at https://doi.org/ 10.1038/s41427-019-0185-z.

Received: 6 August 2019 Revised: 2 October 2019 Accepted: 20 October 2019.

Published online: 20 December 2019

\section{References}

1. Kim, B. \& Mooney, D. Development of biocompatible synthetic extracellular matrices for tissue engineering. Trends Biotechnol. 16, 224 (1998).

2. Kim, S. H. et al. Hydrogel-laden paper scaffold system for origami-based tissue engineering. Proc. Natl Acad. Sci. USA 112, 15426-15431 (2015).

3. Khademhosseini, A. \& Langer, R. Microengineered hydrogels for tissue engineering. Biomaterials 28, 5087-5092 (2007).

4. Fan, Z. et al. A new class of biological materials: cell membrane-derived hydrogel scaffolds. Biomaterials 197, 244-254 (2019).

5. Yu, Y. et al. Nanostructured lipid carrier-based $\mathrm{pH}$ and temperature dualresponsive hydrogel composed of carboxymethyl chitosan and poloxamer for drug delivery. Int. J. Biol. Macromol. 114, 462-469 (2018).

6. Hong, S. et al. Supramolecular metallo-bioadhesive for minimally invasive use. Adv. Mater. 28, 8675-8680 (2016)

7. Wang, K. \& Han, Z. Injectable hydrogels for ophthalmic applications. J. Control. Release 268, 212-224 (2017).

8. Hsieh, F. Y. et al. Non-viral delivery of an optogenetic tool into cells with selfhealing hydrogel. Biomaterials 174, 31-40 (2018).

9. Gan, D. et al. Mussel-inspired contact-active antibacterial hydrogel with high cell affinity, toughness, and recoverability. Adv. Funct. Mater. 29, 1805964 (2019).

10. Derycke, A. S. L. Witte PAMD. Liposomes for photodynamic therapy. Adv. Drug Deliv. Rev. 56, 17-30 (2004)

11. Gradauer, K. et al. Liposomes coated with thiolated chitosan enhance oral peptide delivery to rats. J. Control. Release 172, 872-878 (2013).

12. Kim, K. Kim, K. Ryu, J. H. \& Lee, H. Chitosan-catechol: a polymer with longlasting mucoadhesive properties. Biomaterials 52, 161-170 (2015).

13. Ponchel, G. \& Irache, J. M. Specific and non-specific bioadhesive particulate systems for oral delivery to the gastrointestinal tract. Adv. Drug Deliv. Rev. 34, 191-219 (1998).

14. Lavelle, E. C., Grant, G., Pusztai, A., Pfüller, U. \& O'Hagan, D. T. Mucosal immunogenicity of plant lectins in mice. Immunology 99, 30-37 (2000).

15. Miyake, K, Tanaka, T. \& McNeil, P. L. Lectin-based food poisoning: a new mechanism of protein toxicity. PLoS ONE. 2, e687 (2007).

16. Lee, S. et al. Enhanced therapeutic neovascularization by CD31-expressing cells and embryonic stem cell-derived endothelial cells engineered with chitosan hydrogel containing VEGF-releasing microtubes. Biomaterials $\mathbf{6 3}$, 158-167 (2015).

17. Yue, K. et al. Synthesis, properties, and biomedical applications of gelatin methacryloyl (GelMA) hydrogels. Biomaterials 73, 254-271 (2015).

18. He, Q., Huang, Y. \& Wang, S. Hofmeister effect-assisted one step fabrication of ductile and strong gelatin hydrogels. Adv. Funct. Mater. 28, 1705069 (2018)

19. Cheng, R. et al. Mechanically enhanced lipo-hydrogel with controlled release of multi-type drugs for bone regeneration. Appl. Mater. Today 12, 294-308 (2018).

20. Pérez-San, V. A. et al. Self-healing dynamic hydrogel as injectable shockabsorbing artificial nucleus pulposus. Biomacromolecules 18, 2360-2370 (2017).

21. Cummings, S. R. \& Melton, L. J. Epidemiology and outcomes of osteoporotic fractures. Lancet 359, 1761-1767 (2002).

22. Li, Y., He, S., Hua, Y. \& Hu, J. Effect of osteoporosis on fixation of osseointegrated implants in rats. J. Biomed. Mater. Res. B Appl. Biomater. 105, 2426-2432 (2016).

23. Becker, W, Hujoel, P. P., Becker, B. E. \& Willingham, H. Osteoporosis and implant failure: an exploratory case-control study. J. Periodontol. 71, 625-631 (2000).

24. Barbu, H. M. et al. Dental implant placement in patients with osteoporosis. J. Craniofac. Surg. 26, e558 (2015).
25. Bertoni, S. et al. pH and reactive oxygen species-sequential responsive nanoin-micro composite for targeted therapy of inflammatory bowel disease. Adv. Funct. Mater. 28, 1806175 (2018).

26. Guo, J. et al. Development of tannin-inspired antimicrobial bioadhesives. Acto Biomater. 72, 35-44 (2018).

27. Guo, J. et al. Synthesis and characterization of anti-bacterial and anti-fungal citrate-based mussel-inspired bioadhesives. Biomaterials 85, 204-217 (2016).

28. Tan, L. et al. Rapid biofilm eradication on bone implants using red phosphorus and near-infrared light. Adv. Mater. 30, 1801808 (2018).

29. Li, Y. et al. Eradicating multidrug-resistant bacteria rapidly using a multi functional g-C3N4@Bi2S3 nanorod heterojunction with or without antibiotics. Adv. Funct. Mater. 29, 1900946 (2019).

30. Quan, H. et al. Chemical self-assembly of multi-functional hydroxyapatite with coral-like nanostructure for osteoporotic bone reconstruction. ACS Appl. Mater. Interfaces 10, 25547-25560 (2018).

31. Low, S. A., Galliford, C. V., Jiyuan, Y., Low, P. S. \& Kopeček, J. Biodistribution of fracture-targeted GSK3 $\beta$ inhibitor-loaded micelles for improved fracture healing. Biomacromolecules 16, 3145 (2015).

32. Jia, Z. et al. Simvastatin prodrug micelles target fracture and improve healing. J. Control. Release 200, 23-34 (2015).

33. Hauptstein, S., Dezorzi, S., Prüfert, F., Matuszczak, B. \& Bernkop-Schnürch, A. Synthesis and in vitro characterization of a novel S-protected thiolated alginate. Carbohydr. Polym. 124, 1-7 (2015).

34. Chen, $\mathrm{H}$. et al. The potential use of novel chitosan-coated deformable liposomes in an ocular drug delivery system. Colloids Surf. B Biointerfaces 143, 455-462 (2016).

35. Hoogenboom, R., Schubert, U., Fischer, D. C. \& Knop, F. K. Poly(ethylene glycol) in drug delivery: pros and cons as well as potential alternatives. Angew. Chem. Int. Ed. Engl. 42, 6288-6308 (2011).

36. Lee, A. L. Z., Ng, V. W. L., Gao, S., Hedrick, J. L. \& Yang, Y. Y. Injectable hydrogels from triblock copolymers of vitamin E-functionalized polycarbonate and poly (ethylene glycol) for subcutaneous delivery of antibodies for cancer therapy. Adv. Funct. Mater. 24, 1538-1550 (2014)

37. Dimatteo, R., Darling, N. J. \& Segura, T. In situ forming injectable hydrogels for drug delivery and wound repair. Adv. Drug Deliv. Rev. 127, 167-184 (2018).

38. Li, L., Yan, B., Yang, J., Chen, L. \& Zeng, H. Novel mussel-inspired injectable selfhealing hydrogel with anti-biofouling property. Adv. Mater. 27, 1294-1299 (2015).

39. Tseng, T.-C. et al. An injectable, self-healing hydrogel to repair the central nervous system. Adv. Mater. 27, 3518-3524 (2015).

40. Hsu, R. S. et al. Adaptable microporous hydrogels of propagating NGFgradient by injectable building blocks for accelerated axonal outgrowth. Adv. Sci. 6, 1900520 (2019).

41. Chen, H. et al. An injectable self-healing coordinative hydrogel with antibacterial and angiogenic properties for diabetic skin wound repair. NPG Asia Mater. 11, 3 (2019).

42. Wang, H. et al. Cell-laden photocrosslinked GelMA-DexMA copolymer hydrogels with tunable mechanical properties for tissue engineering. J. Mater. Sci. Mater. Med. 25, 2173-2183 (2014).

43. Ruth, P. et al. Chemical coupling of thiolated chitosan to preformed liposomes improves mucoadhesive properties. Int. J. Nanomed. 7, 2523-2534 (2012).

44. Fan, B. et al. Revealing the assembly mechanism of an octadecylamine based supramolecular complex on mild steel in condensate water: correlative experimental and theoretical studies. J. Mol. Liq. 292, 111446 (2019).

45. Huang, Y., Leobandung, W., Foss, A. \& Peppas, N. A. Molecular aspects of muco- and bioadhesion: tethered structures and site-specific surfaces. J. Control. Release 65, 63-71 (2000).

46. Johnson, C. T. et al. Hydrogel delivery of lysostaphin eliminates orthopedic implant infection by Staphylococcus aureus and supports fracture healing. Proc. Natl Acad. Sci. USA 115, 201801013 (2018).

47. $\mathrm{Xu}$, Z et al. Tannic acid/Fe3 + /Ag nanofilm exhibiting superior photodynamic and physical antibacterial activity. ACS Appl. Mater. Interfaces 9, 39657-39671 (2017).

48. Liu, J., Sonshine, D. A., Shervani, S. \& Hurt, R. H. Controlled release of biologically active silver from nanosilver surfaces. ACS Nano 4, 6903-6913 (2010).

49. He, M., Wang, Q., Zhang, J., Zhao, W. \& Zhao, C. Substrate-independent Agnanoparticle-loaded hydrogel coating with regenerable bactericidal and thermoresponsive antibacterial properties. ACS Appl. Mater. Interfaces 9, 44782-44791 (2017). 
50. Paustovskaia, V. V., Rozhkovskaia, G. P., Rappoport, M. B., Anina, I. A. \& Pasternak, G. A. Biological action of 4,4'-diaminodicyclohexylmethane carbonate and octadecylamine. Gig. Sanit. 38, 31-35 (1973).

51. MacDonald, W. E., Deichmann, W. B., Radomski, J. L. \& Austin, B. S. The chronic toxicity of octadecylamine in the rat-a supplemental report. Toxicol. Appl. Pharmacol. 4, 610-612 (1962).
52. $\mathrm{Xu}, \mathrm{Y}$. et al. A novel lipoprotein-mimic nanocarrier composed of the modified protein and lipid for tumor cell targeting delivery. J. Control. Release 146 299-308 (2010).

53. Kim, H. Y. et al. Sustained release of BMP-2 from porous particles with leafstacked structure for bone regeneration. ACS Appl. Mater. Interfaces 10, 21091-21102 (2018). 